\title{
Cold feet
}

\author{
LARRY G. EPSTEIN \\ Department of Economics, Boston University \\ IGOR KOPYLOV \\ Department of Economics, University of California, Irvine
}

\begin{abstract}
Individuals often lose confidence in their prospects as they approach the 'moment of truth.' An axiomatic model of such individuals is provided. The model adapts and extends (by relaxing the Independence axiom) Gul and Pesendorfer's model of temptation and self-control to capture an individual who changes her beliefs so as to become more pessimistic as payoff time approaches. In a variation of the model, the individual becomes more optimistic at an ex post stage in order to feel better about her available options.
\end{abstract}

KEYWORDS. Temptation, pessimism, non-Bayesian updating, optimism, ambiguity, moment of truth, confidence.

JEL CLASSIFICATION. D81, D83.

\section{INTRODUCTION}

A common finding in psychological studies is that individuals tend to lose confidence in their prospects as they approach the 'moment of truth.' (See Gilovich et al. (1993) and the references therein; for other studies of the connection between confidence and temporal proximity see Armor and Taylor (2002) and Trope and Liberman (2003).) Anecdotal evidence includes students who become more worried about their performance just before taking a test, and 'cold feet' on a wedding day. We provide a choice-theoretic model of such agents. Since we assume that beliefs are observable only indirectly through choice, our focus is on the question: what behavior would reveal increasing pessimism as the moment of truth becomes imminent?

Though it is most often described as modeling ambiguity aversion, the multiplepriors model of Gilboa and Schmeidler (1989) is sometimes interpreted in terms of pessimism, since the agent chooses an action as though she first chooses the least favorable prior in her set of priors. We model pessimism similarly via minimization over a set of probability measures. However, our model differs from that of Gilboa and Schmeidler

Larry G. Epstein: lepstein@bu . edu

Igor Kopylov: ikopylov@uci . edu

Epstein gratefully acknowledges the financial support of the NSF (award SES-0611456). We have benefited also from comments of Eddie Dekel, Faruk Gul, Bart Lipman, Fabio Maccheroni, Massimo Marinacci, Jawwad Noor, Wolfgang Pesendorfer, three referees, and especially Jeff Ely. This paper is a substantial revision of our earlier paper titled 'Cognitive Dissonance and Choice.'

Copyright (c) 2007 Larry G. Epstein and Igor Kopylov. Licensed under the Creative Commons AttributionNonCommercial License 2.5. Available at http: //econtheory . org. 
in that its focus is on time-varying pessimism, while their model is static. It differs also from the dynamic version of multiple-priors proposed by Epstein and Schneider (2003), because only here does time-varying pessimism lead to a demand for commitment. The latter arises much as in the Gul and Pesendorfer (2001) model of temptation and self-control, which we adapt to model preference and behavior given time-varying pessimism. Thus formally our model combines Gilboa-Schmeidler and Gul-Pesendorfer (henceforth GP).

A brief outline follows. Uncertainty is represented by the (finite) state space $S$, and time varies over three periods. The true state is realized and payoffs are received at the terminal time. A physical action is chosen at the initial ex ante stage. Each such action is modeled by a menu of (Anscombe-Aumann) acts-the idea is that any action taken ex ante limits options ex post. The agent understands when choosing a menu that at an intermediate time, called the ex post stage, she will choose an act from that menu. In addition, she anticipates that the passage of time will have an effect on her outlook. Ex ante, with the objectivity afforded by temporal distance, she is cool-headed and adopts beliefs that seem 'correct'-they are represented by a Savage prior on $S$. Ex post, she is closer to the moment of truth; anxiety about unfavorable outcomes increases and leads to pessimism about the prospects for the previously chosen menu-she chooses a new belief about $S$ that makes the menu less attractive. ${ }^{1}$ She is tempted to choose out of the menu by maximizing expected utility using the new pessimistic belief. However, there is a tension because she recognizes that her prior beliefs, having been formed at a stage where she was more cool-headed and clear-sighted, would be a better guide for the decisions still to be made. Thus she may exert costly self-control. Regardless, temptation is costly, and anticipation of these costs affects her ranking of menus-for example, she may prefer a smaller menu, or even a singleton that would commit her to a fixed action ex post, in order to reduce the costs of temptation and self-control. Thus the ex ante ranking of menus reveals her expected change in beliefs.

Three remarks about the model merit emphasis. First, by 'pessimism' we have in mind an agent who has a negative picture of the future regardless of the prospect she is holding. This cannot be modeled simply by assuming subjective expected utility maximization with a 'pessimistic probability measure'-any change in beliefs will make some prospects look less attractive but will render others more attractive. Essentially, the 'pessimistic belief' must vary with the prospect in hand, and this is achieved endogenously in our model.

Second, our agent is not boundedly rational. Since there are no objective probabilities available to describe likelihoods of states in $S$, or undeniable facts that pin down 'correct' beliefs, it is not irrational to change one's subjective assessment as time passes, even in the absence of new information. Moreover, our agent is sophisticated

\footnotetext{
${ }^{1}$ The assumption of correct beliefs only at the ex ante stage is consistent in spirit with the broader idea that the greater is the temporal distance from the consequences of choice, the more choice conforms to 'true' or 'normative' preferences. Supporting evidence includes findings on so-called 'preference reversals' and dynamic inconsistency. See Frederick et al. (2002), for example; see also Noor (2007) for elaboration and for a model of temptation based on the idea that normative preference is revealed by the ranking of temporally distant alternatives.
} 
and forward-looking - when choosing an action ex ante she is fully aware that she may develop cold feet as the moment of truth approaches. She has this sophistication in common with agents in most economic models, but one may wonder whether individuals outside those models are typically self-aware to this degree. We are not familiar with definitive evidence on this question, and in its absence we are inclined to feel that full self-awareness is a plausible working hypothesis. ${ }^{2}$ Even where the opposite extreme of complete naivete seems descriptively more accurate, our model may help to clarify which economic consequences are due to time-varying pessimism per se and which are due to naivete. In addition, the assumption of sophistication is vital for a choicetheoretic approach: because she anticipates her future pessimism, it affects her current choice of actions. This makes it possible to infer her expected pessimism from her (in principle observable) choice of actions, consistent with the choice-theoretic tradition of Savage. Thus sophistication seems justifiable also on the methodological ground of permitting the exploration of modest departures from standard models.

Finally, some readers may wonder about the connection between (costly) selfcontrol and cold feet. It is intuitive to us that when tempted to deviate from plans based on ex ante clear-thinking beliefs, and while recalling those, different agents will exhibit self-control to different degrees, including some who may exert no self-control. Thus one can readily imagine cold feet without self-control. ${ }^{3}$ However, though self-control is not intrinsic to cold feet, its absence seems to us to be a boundary case: in our model self-control is parametrized by a positive parameter $\kappa$, and approaches zero as $\kappa \nearrow \infty$. GP (p. 1412) explain in their model the sense in which preferences without self-control can be viewed informally as a 'limit' of preferences with self-control, and they show how their formal analysis can be extended, at the cost of relaxing and complicating the continuity property assumed for preference, to include these limiting preferences. We suspect that we could do the same here, though we have not pursued such an extension because we do not view the boundary case as particularly important.

We conclude this introduction with an elaboration of the connections to the literature. Though we adapt GP, their model does not apply directly. One difference is that while they study preferences over menus of lotteries, it is important for our story that menus consist of (Anscombe-Aumann) acts. This is because, while GP are concerned with temptation that arises from a change in taste (ranking of lotteries), our story is that it is a change in subjective beliefs that is the source of temptation. Epstein (2006) shows that GP's temptation model can be adapted to address belief distortions (he makes the point in the context of a model of non-Bayesian updating). In a more technical vein, Kopylov (2005) extends the GP theorem from (menus of) lotteries to abstract mixture spaces, including, in particular, the space of Anscombe-Aumann acts.

\footnotetext{
${ }^{2} \mathrm{~A}$ range of behavior might be understood in part as reflecting (partial) commitment motivated by the expectation of future pessimism. Widely announced and elaborately celebrated engagements make it costly to succumb to cold feet at the wedding altar. An example in a more standard economic setting is the growing tendency for investors to delegate investment decisions.

${ }^{3}$ In fact, one could imagine also the opposite of costly self-control—satisfaction from overcoming temptation. A general model would permit also such a reaction to ex post pessimism. We focus on costly selfcontrol because we find it more intuitive.
} 
However, all these papers adopt the Independence axiom (appropriate for their respective settings), while we relax Independence significantly. Perhaps not surprisingly, given Gilboa and Schmeidler's model, we argue that Independence is not intuitive in a model of pessimism, specifically, where the (ex post) distortion of beliefs depends on the set of available options. Permitting such a dependence constitutes a significant improvement over Epstein's model of beliefs distortion (see Section 5 for further discussion). At the same time, our relaxation of Independence is the major source of technical difficulty and novelty in our model-it necessitates novel proofs rather than adaptations of GP's arguments.

Finally, we note that Dekel et al. (2006) generalize GP's model of temptation. However, their motivation is much different from ours—in particular, they assume Independence. $^{4}$

The paper proceeds as follows. The model's primitives and the functional form for utility are described in the next section. Then Section 3 describes its underlying axiomatic foundations. Section 4 contains our main representation result, as well as some comparative behavioral characterizations that facilitate interpretations of the components of the functional form for utility. Section 5 concludes by discussing a variation of the model concerning time-varying optimism (as opposed to pessimism), and by outlining an extension that models how pessimistic (or optimistic) agents respond to information.

\section{UTILITY}

The model has the following primitives.

- Time $t=0,1,2$.

- Finite state space $S$.

- $\mathscr{C}$ : set of (Borel) probability measures over a compact metric space. We refer to $c \in \mathscr{C}$ as a lottery over consumption, or more briefly as consumption. The set $\mathscr{C}$ is compact metric under the weak convergence topology.

- $\mathscr{H}$ : set of acts $h: S \longrightarrow \mathscr{C}$, with the usual mixture operation.

- Compact sets of acts are called menus and denoted $A, B, \ldots . \mathscr{K}(\mathscr{H})$ is the set of all menus; it is compact metric under the Hausdorff metric. ${ }^{5}$

- Preference $\succeq$ defined on $\mathscr{K}(\mathscr{H})$.

\footnotetext{
${ }^{4}$ In their concluding remarks about possible directions for further research, they mention that accommodating guilt may be a reason for relaxing Independence when modeling temptation. This rationale is obviously much different from ours. There exist other representation results in the menus-of-lotteries/acts setting that do not rely on Independence. Epstein et al. (forthcoming) study an agent who is not subject to temptation, but rather values flexibility because she is uncertain about the future; she violates Independence because her conception of the future is coarse. Other results, with still different objectives, appear in Ergin and Sarver (2005) and Noor (2006).

${ }^{5}$ See Aliprantis and Border (1994, Theorem 3.58), for example.
} 
The interpretation is that a menu $A$ is chosen ex ante (at time 0 ) according to $\succeq$. This choice is made with the understanding that at the unmodeled ex post stage (time 1), the agent will choose an act from $A$. Uncertainty is resolved and consumption is realized in the terminal period $t=2$. Cold feet, pessimism, and choice behavior at time 1 are anticipated ex ante and underlie the ranking $\succeq$.

Menus are natural objects of choice ${ }^{6}$ The consequence of a physical action taken at time 0 is that it determines a feasible set of physical actions at time 1 , and these actions can be modeled by acts in the usual way. Thus each physical action at time 0 corresponds to a menu of acts.

Our model of utility has the form ${ }^{7}$

$$
\mathscr{U}(A)=\max _{h \in A}[U(h)+\kappa V(h)]-\kappa \max _{h^{\prime} \in A} V\left(h^{\prime}\right),
$$

where

$$
\begin{gathered}
U(h)=p \cdot u(h) \\
V(h)=\min _{q \in Q} q \cdot u(h) .
\end{gathered}
$$

Here $\kappa \geq 0, p$ is a probability measure on $S, Q$ is a closed and convex set of probability measures on $S$ containing $p$, and $u: \mathscr{C} \longrightarrow \mathbb{R}^{1}$ is mixture linear and continuous.

The standard model of subjective expected utility maximization is the special case where $\kappa=0$ or $Q=\{p\}$. More generally, the functional form can be interpreted along the lines suggested by GP. When restricted to singletons, $\mathscr{U}$ coincides (ordinally) with $U$; thus expected utility with prior $p$ represents preference over consumption lotteries when the agent can commit ex ante. The ex ante stage is far enough removed from payoff time that the agent is collected, and $p$ represents her clear-thinking view of likelihoods. However, if she chooses a menu $A$ that does not provide commitment, then she anticipates the following: she knows that ex post, near the moment of truth, she will be more nervous about the possible unfavorable outcomes and she will exaggerate their likelihoods-this is captured by minimization over the set $Q$ (since $p \in Q$, minimization over $Q$ imputes lower expected utility to any act than was the case ex ante using $p$ ). Anxiety creates the temptation to maximize $V$ ex post. However, she may resist since she is aware that $p$ is a better guide for remaining decisions. The cost of controlling her feelings of anxiety and resisting the temptation is given by ${ }^{8}$

$$
\kappa\left(V(h)-\max _{h^{\prime} \in A} V\left(h^{\prime}\right)\right) \leq 0 .
$$

\footnotetext{
${ }^{6}$ Kreps $(1979,1992)$ was the first to propose menus as a way to model physical actions in an ex ante stage.

${ }^{7}$ For any real-valued random variable $x$ on $S$ and probability measure $q, q \cdot x$ is shorthand for the expected value $\int_{S} x d q$.

${ }^{8}$ In the limit as $\kappa \rightarrow \infty$, the cost of deviating from maximization of $V$ becomes prohibitive and the model suggests the Strotz-like utility function

$$
\mathscr{U}(A)=\max _{h \in A}\left\{U(h): V(h)=\max _{h^{\prime} \in A} V\left(h^{\prime}\right)\right\} .
$$

As mentioned in the introduction, this limiting model is excluded from our formal analysis.
} 
Thus a compromise is struck between maximizing $U$ and maximizing $V$ and choice out of $A$ is described by maximization of the weighted sum, or by solving

$$
\max _{h \in A} \min _{q \in \frac{1}{1+\kappa}\{p\}+\frac{\kappa}{1+\kappa} Q} q \cdot u(h),
$$

which balances the ex ante view $p$ and ex post pessimism. The nature of the compromise is further illustrated by the fact that

$$
p \in \frac{1}{1+\kappa}\{p\}+\frac{\kappa}{1+\kappa} Q \subseteq Q
$$

so that the set of beliefs underlying the choice of an act ex post lies 'between' the prior view $p$ and the pessimistic view represented by $Q .{ }^{9}$ Note that since this compromise is motivated exclusively by self-control costs rather than by the arrival of new information or by a more sophisticated ex post assessment of probabilities, the parameter $\kappa$ determining the compromise belief is naturally the same one appearing in the expression (4) for the self-control cost.

Note that both subjective and objective probabilities are present in the model-the latter underlie consumption lotteries-but they are treated differently: while the agent chooses new beliefs ex post about her subjective uncertainty (the state space $S$ ), she does not distort or modify objective probabilities. For example, both $U$ and $V$ agree about the ranking of lotteries in that, for every lottery $c, U(c)=V(c)=u(c)$, the vNM expected utility of $c$. Therefore the agent in our model is free to choose beliefs and to change them as the moment of truth approaches, but only if these beliefs are not pinned down uniquely by objective facts.

\section{Axioms}

The first two axioms require no discussion.

AxIOM 1 (Order). $\succeq$ is complete and transitive.

Aхіом 2 (Continuity). $\succeq$ is continuous.

Menus can be mixed via

$$
\alpha A+(1-\alpha) B=\{\alpha f+(1-\alpha) g: f \in A, g \in B\} .
$$

Formally, the indicated mixture of $A$ and $B$ is another menu and thus when the agent contemplates that menu ex ante, she anticipates choosing out of $\alpha A+(1-\alpha) B$ ex post. It follows that one should think of the randomization corresponding to the $\alpha$ and $(1-\alpha)$ weights as taking place at the end-after she has chosen some mixed act $\alpha f+(1-\alpha) g$ out of the menu. In fact, since the mixture of acts is defined by $(\alpha f+(1-\alpha) g)(s)=$ $\alpha f(s)+(1-\alpha) g(s)$ for each $s$, the randomization occurs after the realization of the state.

\footnotetext{
${ }^{9} \mathrm{As}$ is familiar from GP-style models, this interpretation in terms of ex post choice is suggested by the functional form, and by intuition for the underlying axioms, but ex post choice lies outside the scope of our formal model. See Noor (2007) for a model of temptation where ex post choice is part of the primitives.
} 
The above mixture operation permits one to state the Independence axiom, which is adopted by GP. However, Independence is not intuitive under ex post pessimism. ${ }^{10}$ To see this, suppose for concreteness that $A \sim B$ and consider whether the mixture $\alpha A+(1-\alpha) B$ should also be indifferent to $A$ as required by Independence. Indifference between $A$ and $B$ is based on the anticipation that, in each case, the agent will choose beliefs ex post to make the menu in hand unattractive. Evaluation of the mixture $\alpha A+(1-\alpha) B$ can be thought of similarly, but the important point is that beliefs for the mixed menu must be chosen before the randomization is played out. Since also beliefs chosen given A generally differ from those chosen given $B$, pessimistic beliefs for the mixed menu bear no simple relation to those for $A$ and $B$. A similar disconnect applies to anticipated temptation and ex post choices across the three menus. For example, it is possible that the acts $f$ and $g$ be chosen out of $A$ and $B$ respectively, while $\alpha f+(1-\alpha) g$ not be chosen out of $\alpha A+(1-\alpha) B$. As a result, the agent will generally not be indifferent between the mixed menu and $A$, violating Independence. (The deviation from indifference could go in either direction: $\alpha A+(1-\alpha) B \succ A$ and $\alpha A+(1-\alpha) B \prec A$ are both possible.)

To illustrate how the anticipation of ex post pessimism can lead to a violation of Independence, let $E \subset S$ be an event and let $f$ denote the bet on $E$ paying $\$ 100$ if the realized state lies in $E$ and $\$ 0$ otherwise. If, according to her cool-headed prior, $E$ is strictly more likely than its complement $S \backslash E$, the agent would strictly prefer $f$ ex ante to the constant act $c$ that yields a lottery with equiprobable payoffs $\$ 100$ and $\$ 0$. However, given the option to switch to $c$ ex post, pessimism at that stage about the likelihood of $E$ may lead to the temptation to do so. Anticipating this, she would prefer to commit to $f$ ex ante, and thus would exhibit the ranking

$$
\{f\} \succ\{f, c\} \succeq\{c\} .
$$

However, the strict preference for commitment may be problematic if $f$ and $c$ are each mixed with a common third bet $g$, contrary to Independence. For example, let $g$ be the bet on $S \backslash E$ given by $g=\left(\$ 0\right.$ if $E ; \$ 100$ if $S \backslash E$ ). (Note that $\frac{1}{2} f+\frac{1}{2} g=c$.) Ex ante, since $E$ is viewed as more likely than its complement, the commitment ranking

$$
\{c\}=\left\{\frac{1}{2} f+\frac{1}{2} g\right\} \succ\left\{\frac{1}{2} c+\frac{1}{2} g\right\}
$$

is natural. However, since $g$ hedges $f$ perfectly, ex post pessimism does not affect the evaluation of the mixture $\frac{1}{2} f+\frac{1}{2} g$, and there is no temptation to choose $\frac{1}{2} c+\frac{1}{2} g$ when the latter is available. Therefore,

$$
\left\{\frac{1}{2} f+\frac{1}{2} g\right\} \sim\left\{\frac{1}{2} f+\frac{1}{2} g, \frac{1}{2} c+\frac{1}{2} g\right\} .
$$

In this way, hedging can eliminate a demand for commitment, contrary to Independence.

\footnotetext{
${ }^{10}$ The reason is essentially that because the agent anticipates that she will adjust her beliefs ex post to the menu at hand, the situation is analogous to that of choice between 'temporal risks.' As explained by Machina (1984), for example, preferences over temporal risks typically violate Independence even at a normative level.
} 
However, suitable relaxations of Independence are intuitive. When ranking singleton menus, there is no choice to be made ex post. Thus ex post beliefs are not relevant and there is no reason for Independence to be violated. This motivates the following relaxation of Independence.

AxIom 3 (Commitment Independence). For all $f, g, h \in \mathscr{H}$ and $\alpha \in(0,1)$,

$$
\{f\} \succ\{g\} \Longrightarrow\{\alpha f+(1-\alpha) h\} \succ\{\alpha g+(1-\alpha) h\}
$$

To formulate a second relaxation of Independence, adopt some notation. For any act $f \in \mathscr{H}$, let

$$
\mathscr{H}_{f}=\{t c+(1-t) f: t \in[0,1], c \in \mathscr{C}\} .
$$

If $h=t c+(1-t) f$ is an act in $\mathscr{H}_{f}$, then for any mixture linear $u$ and for all probability measures $q$,

$$
q \cdot u(h)=t u(c)+(1-t) q \cdot u(f) .
$$

Because the first term on the right is independent of $q$, it follows that any menu $A$ that is a subset of $\mathscr{H}_{f}$ is rendered unattractive by beliefs that make $f$ unattractive. In particular, for any two menus $A$ and $B$ in $\mathscr{H}_{f}$, when the agent chooses beliefs to fit the menu, there is a pessimistic measure that is common to both $A$ and $B$. But this invalidates the reason given above for violating Independence. Thus we adopt the following axiom.

Aхıом 4 (Collinear Independence). For all $\alpha \in(0,1)$, for all $f \in \mathscr{H}$, and for all menus $A^{\prime}$, $A, B \subseteq \mathscr{H}_{f}$,

$$
A^{\prime} \succ A \Longrightarrow \alpha A^{\prime}+(1-\alpha) B \succ \alpha A+(1-\alpha) B .
$$

Acts $h^{\prime}$ and $h$ in $\mathscr{H}_{f}$ are naturally called collinear, which explains the name of the axiom. ${ }^{11}$

In the standard model, a menu is as good as the best alternative that it contains. Then

$$
A \succeq B \Longrightarrow A \sim A \cup B,
$$

a property called strategic rationality by Kreps (1988). Such a model excludes temptation. Temptation is an integral part of time-varying pessimism because the agent changes beliefs to make the menu at hand look unattractive and then is tempted to make subsequent choices accordingly (see the discussion of utility in Section 2). In seeking a suitable relaxation of strategic rationality, we begin with GP's central axiom Set-Betweenness.

Set-Betweenness (SB). For all menus $A$ and $A^{\prime}$, if $A \succeq A^{\prime}$, then $A \succeq A \cup A^{\prime} \succeq A^{\prime}$.

\footnotetext{
${ }^{11}$ For any collinear acts $h^{\prime}$ and $h$, it is easy to see that for every $s^{\prime}$ and $s, u\left(h^{\prime}\left(s^{\prime}\right)\right)>u\left(h^{\prime}(s)\right)$ implies $u\left(h\left(s^{\prime}\right)\right) \geq u(h(s))$, that is, the real-valued functions $u(h(\cdot))$ and $u\left(h^{\prime}(\cdot)\right)$ are comonotonic. Collinearity implies the stronger restriction $(1-t)\left(u\left(h^{\prime}\left(s^{\prime}\right)\right)-u\left(h^{\prime}(s)\right)\right)=\left(1-t^{\prime}\right)\left(u\left(h\left(s^{\prime}\right)\right)-u(h(s))\right)$ for some $t$ and $t^{\prime}$ and all $s$ and $s^{\prime}$. Thus collinearity can be viewed as a cardinal counterpart of comonotonicity.
} 
An equivalent and perhaps more revealing, though less compact, statement is that if $A \succeq A^{\prime}$, then one of the following conditions holds: (i) $A \sim A \cup A^{\prime} \sim A^{\prime}$, (ii) $A \succ A \cup A^{\prime} \succ A^{\prime}$, (iii) $A \sim A \cup A^{\prime} \succ A^{\prime}$, or (iv) $A \succ A \cup A^{\prime} \sim A^{\prime}$.

Following GP (p. 1408), we may interpret these conditions intuitively. The underlying assumptions are that: unchosen acts can only reduce utility, acts can be ranked according to how tempting they are, and only the most tempting act affects utility. Consider an agent having the menu $A \cup A^{\prime}$ who expects to choose $f$ though she finds $g$ most tempting. Case (i) is the residual case. Case (ii) indicates that $g$ is in $A^{\prime}$ (hence $A \succ A \cup A^{\prime}$ ) and that $f$ is in $A$ (hence $A \cup A^{\prime} \succ A^{\prime}$ ). The next two cases are our main interest.

In (iii), she still plans to choose out of $A$, which now contains also the most tempting act. Confront her next with the larger menu $A \cup A^{\prime} \cup B$. The most tempting act lies in $A \cup B$. What about her choice out of $A \cup A^{\prime} \cup B$ ? Suppose that her expected choices satisfy the Nash-Chernoff condition (or Sen's property $\alpha$ ); defer for the moment discussion of possible objections to this assumption. Then having rejected acts in $A^{\prime}$ when facing $A \cup A^{\prime}$, she would (expect to) reject them also when facing $A \cup A^{\prime} \cup B$. Thus $A \cup B$ contains both the act to be chosen and also the act in $A \cup A^{\prime} \cup B$ that is most tempting. The indifference $A \cup B \sim A \cup A^{\prime} \cup B$ follows.

Finally, consider (iv), which indicates that both $f$ and $g$ lie in $A^{\prime}$. Again confront the agent with the larger menu $A \cup A^{\prime} \cup B$. The most tempting act lies in $B \cup A^{\prime}$ and, assuming the Nash-Chernoff condition, so does the act to be chosen. Deduce the indifference $A^{\prime} \cup B \sim A \cup A^{\prime} \cup B$.

The preceding provides intuition for the following axiom.

AxIOM 5 (Strong Set-Betweenness (SSB)). For all menus $A$ and $A^{\prime}$, if $A \succeq A^{\prime}$, then

(i) $A \sim A \cup A^{\prime} \sim A^{\prime}$, or

(ii) $A \succ A \cup A^{\prime} \succ A^{\prime}$, or

(iii) $A \sim A \cup A^{\prime} \succ A^{\prime}$ and $A \cup B \sim A \cup A^{\prime} \cup B$ for all menus $B$, or

(iv) $A \succ A \cup A^{\prime} \sim A^{\prime}$ and $A^{\prime} \cup B \sim A \cup A^{\prime} \cup B$ for all menus $B$.

Obviously SSB implies Set-Betweenness. We show below that SSB is strictly stronger than Set-Betweenness even given all our other axioms. However, the two axioms are equivalent given Independence (and Order and Continuity) - this follows from counterparts of the representation results in Gul and Pesendorfer (2001) and Kopylov (2005) and thus SSB is satisfied in their models even though it is not invoked explicitly.

Our intuition for SSB assumes the Nash-Chernoff condition, which can be criticized in a model of temptation-the addition of the acts in $B$ should not affect the normative appeal of $A$ versus $A^{\prime}$, but it may change the self-control costs associated with various choices, and this may lead to the choice of an act in $A^{\prime}$ when facing $A \cup B \cup A^{\prime}$ even where the agent chooses an act in $A$ when facing $A \cup A^{\prime}$. GP's rationale (Theorem 5) for Set-Betweenness also relies on Nash-Chernoff, at least implicitly; see specifically their Axiom T1, which states that choosing an alternative from a menu $A$ is always at least as good as choosing it from a larger menu. 
The Nash-Chernoff condition is weaker than the weak axiom of revealed preference (WARP), which requires also Sen's condition $\beta$ (see Kreps 1988). Noor (2007) provides an example to illustrate why WARP may be problematic in a model of temptation, and Noor (2006) develops a model of temptation and self-control that does not impose WARP for ex post choice. Such objections apply also to the GP model since SSB and WARP for ex post choice are implied when one assumes Independence. Moreover, while they may be important for guiding development of a general model of temptation, these concerns do not seem germane to our focus here.

One can raise other objections to Set-Betweenness, and hence $a$ fortiori to our stronger axiom. Dekel et al. (2006) argue that Set-Betweenness excludes some forms of temptation, for example, where the temptation generated by different alternatives is cumulative, or where there is uncertainty ex ante about which alternatives will be tempting. Once again, we do not view these concerns as especially important for a model of pessimism.

Say that $f \in \mathscr{H}$ dominates $g \in \mathscr{H}$ if $\{f(s)\} \succeq\{g(s)\}$ for every $s \in S$. If the evaluation of a lottery does not depend on the state, then a dominating act should be preferred under commitment. Similarly, if $f$ dominates $g$, we would not expect $f$ to be tempted by $g$. Thus we make the following assumption.

AXIOM 6 (Monotonicity). If $f$ dominates $g$, then $\{f\} \sim\{f, g\} \succeq\{g\}$.

Our axioms thus far have for the most part been concerned with modeling temptation in general, that is, they are not tied specifically to time-varying pessimism. A partial exception is Collinear Independence, the intuition for which relies on the assumption that temptation arises because of an ex post choice of beliefs to 'fit the menu' in hand. However, Collinear Independence is satisfied even if the agent becomes more optimistic ex post and adopts beliefs that make the menu more attractive ex post. The final two axioms build in ex post pessimism.

Axiom 7 (Constants-Cannot-Be-Tempted). For all $c \in \mathscr{C}$ and $f \in \mathscr{H}$,

$$
\{c\} \succ\{f\} \Longrightarrow\{c\} \sim\{c, f\} .
$$

Temptation is due to a change in beliefs (as opposed to a change in risk aversion, for example), which leaves the evaluation of constant acts unaffected. In addition, the noted change is always to become more pessimistic ex post about the available menu, rendering it even less attractive relative to any constant act $c$ than it was ex ante. Therefore, constant acts cannot be tempted. Note that, in contrast, $\{f\} \succ\{c, f\} \succeq\{c\}$ is both permitted by the axiom and intuitive given our story.

The axiom distinguishes our story about the reason for temptation from GP's. The intuition given for the axiom relies on beliefs, but not tastes (the ranking of constant acts), changing between the ex ante and ex post stages. This rules out underlying temptations of the sort considered by GP (for example, the temptation to eat french fries rather than broccoli). 
AxIOM 8 (Concave Temptation). The set $\{f \in \mathscr{H}:\{f\} \sim\{f, c\} \succ\{c\}\}$ is convex for every $c \in \mathscr{C}$.

Suppose that $f$ and $g$ both lie in the indicated set, that is, each is better than $c$ under commitment and not tempted by $c$. Consider the mixture $\alpha f+(1-\alpha) g$. By Commitment Independence, $\{\alpha f+(1-\alpha) g\} \succ\{c\}$. We now argue that, in addition, $c$ should not tempt $\alpha f+(1-\alpha) g$, thus completing intuition for the axiom. We are given that $\{f\} \sim$ $\{f, c\} \succ\{c\}$. Because ex post beliefs are chosen to make the menu $\{f, c\}$ unattractive, and because the expected utility of $c$ does not depend on beliefs, we can interpret the indicated strict preference as follows: the act $f$, even when matched with the beliefs that make it unattractive, is better than $c$. A similar statement applies for $g$. Consider now the menu $\{\alpha f+(1-\alpha) g, c\}$. Beliefs to render this menu unattractive are chosen ex post (time 1), before the randomization is completed (which, as noted earlier, occurs only at the terminal time after the true state in $S$ is realized). Since the beliefs that make $f$ unattractive may differ from those that make $g$ unattractive, matching beliefs with the mixed act is more difficult. Therefore, one would expect $c$ not to tempt $\alpha f+(1-\alpha) g$. Since the set of non-tempted acts is convex, we use the name Concave Temptation. ${ }^{12}$

\section{REPRESENTATION RESUlT}

Our main result is that the preceding axioms characterize the functional form described in Section 2.

THEOREM 1. The binary relation $\succeq$ on $\mathscr{K}(\mathscr{H})$ may be represented as in (1)-(3) if and only if it satisfies Axioms 1-8. Moreover, $u$ is unique up to a positive linear transformation, and if $\succeq$ is not strategically rational, then $p, Q$, and $\kappa$ are unique.

If $Q=\{p\}$ is a singleton or $\kappa=0$, then the preference $\succeq$ is strategically rational and satisfies Independence. The non-degenerate case in the theorem is obtained if $Q$ is a non-singleton and $\kappa>0$. Then $\succeq$ violates both strategic rationality and Independence, and the components $p, Q$, and $\kappa$ in the representation (1)-(3) are unique.

Note that Concave Temptation is used only at the very end of the sufficiency proof in order to obtain the form (3) for the temptation utility $V$. If the axiom is deleted, then the remaining axioms characterize the representation (1)-(2), for some $V: \mathscr{H} \rightarrow \mathbb{R}^{1}$ that is continuous, monotone $(V(f) \geq V(g)$ if $f$ dominates $g)$, satisfies certainty additivity $(V(\alpha f+(1-\alpha) c)=\alpha V(f)+(1-\alpha) V(c)$ for all $c$ in $\mathscr{C})$, and satisfies $V(f) \leq p \cdot u(f)$ for all $f$ with equality if $f$ is constant. (See Example 3 below.)

We present some examples to demonstrate the tightness of the characterization in the theorem. Each of the first three examples satisfies Order, Continuity, Commitment Independence, Strong Set-Betweenness, and Monotonicity, and violates precisely one of the axioms that relate more specifically to pessimism-Collinear Independence, Constants-cannot-be-tempted and Concave Temptation. The final example violates

\footnotetext{
${ }^{12}$ Think of a function defined on $\mathbb{R}^{n}$, and the fact that every upper contour set is convex if and only if the function is quasi-concave.
} 
only Strong Set-Betweenness, though it satisfies GP's Set-Betweenness, thus proving that our adoption of the stronger axiom is necessary.

EXAMPLE 1. Let

$$
\mathscr{U}(A)=\frac{w(A)}{v(A)} \equiv \frac{\max _{h \in A}[U(h) V(h)]}{\max _{h^{\prime} \in A} V\left(h^{\prime}\right)},
$$

where $U$ and $V$ are as in (2)-(3) and $u>0$. Then $\succeq$ violates only Collinear Independence. In particular, to verify SSB note that for any menus $A$ and $A^{\prime}$, there are only four possible cases:

(i) $w(A)>w\left(A^{\prime}\right)$ and $v\left(A^{\prime}\right)>v(A)$; then $\mathscr{U}(A)>\mathscr{U}\left(A \cup A^{\prime}\right)>\mathscr{U}\left(A^{\prime}\right)$.

(ii) $w\left(A^{\prime}\right)>w(A)$ and $v(A)>v\left(A^{\prime}\right)$; then $\mathscr{U}\left(A^{\prime}\right)>\mathscr{U}\left(A \cup A^{\prime}\right)>\mathscr{U}(A)$.

(iii) $w(A) \geq w\left(A^{\prime}\right)$ and $v(A) \geq v\left(A^{\prime}\right)$; then for all menus $B, w(A \cup B)=w\left(A \cup A^{\prime} \cup B\right)$, $v(A \cup B)=v\left(A \cup A^{\prime} \cup B\right)$, and hence $\mathscr{U}(A \cup B)=\mathscr{U}\left(A \cup A^{\prime} \cup B\right)$.

(iv) $w\left(A^{\prime}\right) \geq w(A)$ and $v\left(A^{\prime}\right) \geq v(A)$; then analogously to (iii), $\mathscr{U}\left(A^{\prime} \cup B\right)=\mathscr{U}\left(A \cup A^{\prime} \cup B\right)$ for all menus $B$.

There exist simpler examples violating only Collinear Independence-these retain (1)-(2) but modify the specification of $V$. However, because the above ratio form deviates from the GP functional form, we find it more revealing about the power of Collinear Independence. ${ }^{13}$

EXAMPLE 2. Assume (1)-(2), but take

$$
V(h)=q \cdot u(h)
$$

for some probability measure $q \neq p$. Then $\succeq$ violates only Constants-Cannot-BeTempted.

EXAMPLE 3. Modify Example 2 by taking

$$
V(h)=\min \left\{p \cdot u(f), \int u(f) d v\right\},
$$

where $v$ is a capacity on $S$ and the integral $\int u(f) d v$ is in the sense of Choquet (see Schmeidler 1989). Then $\succeq$ violates only Concave Temptation.

\footnotetext{
${ }^{13}$ The example is inspired by weighted utility theory (Chew 1983), a model of risk preference in which the utility function over lotteries equals a ratio of expected utility functions. Readers familiar with the 'nonexpected utility' literature will not be surprised by the observation that $\succeq$ satisfies the following alternative relaxation of Independence: $A \sim B \Longrightarrow \alpha A+(1-\alpha) B \sim A$.
} 
EXAMPLE 4. This example violates Strong Set-Betweenness, but satisfies all other axioms adopted in Theorem 1 as well as GP's Set-Betweenness. Let $S=\left\{s_{1}, s_{2}\right\}$, and fix a vNM utility function $u$ such that $u(\mathscr{C})=[0,1]$. For every act $f \in \mathscr{H}$ and menu $A$, let $u_{1}(f)=u\left(f\left(s_{1}\right)\right), u_{2}(f)=u\left(f\left(s_{2}\right)\right)$, and

$$
\begin{gathered}
\gamma(f)=\max \left\{0, u_{2}(f)-u_{1}(f)-\frac{4}{5}\right\} \\
v(f)=\max \left\{0, u_{1}(f)-u_{2}(f)-\frac{4}{5}\right\} \\
\mathscr{U}(A)=\max _{f \in A}\left[u_{2}(f)-\gamma(f) \max _{g \in A} v(g)\right] .
\end{gathered}
$$

Let $\succeq$ be represented by $\mathscr{U}$. Then $\succeq$ obeys Order and Continuity. Note that

$$
\begin{array}{lllll}
\gamma(f)>0 & \Longrightarrow & u_{1}(f)<\frac{1}{5} & \text { and } & u_{2}(f)>\frac{4}{5} \\
v(g)>0 & \Longrightarrow & u_{1}(g)>\frac{4}{5} & \text { and } & u_{2}(g)<\frac{1}{5} .
\end{array}
$$

Therefore, $\gamma(f) v(f)=0$ for all $f$, so that $\mathscr{U}(\{f\})=u_{2}(f)$, implying Commitment Independence. In addition, $\gamma(f) v(g)=0$ holds in each of the following cases: (i) $f$ or $g$ is constant; (ii) $f$ and $g$ are collinear; (iii) $f$ dominates $g$; (iv) $g$ dominates $f$. Thus $\succeq$ satisfies Collinear Independence, Monotonicity, Constants-cannot-be-tempted, and Concave Temptation (because constants are not tempting in this example). For SetBetweenness, take any menus $A$ and $B$ and acts $f, g \in A \cup B$ that deliver the maxima in the definition of $\mathscr{U}(A \cup B)$, so that $\mathscr{U}(A \cup B)=u_{2}(f)-\gamma(f) v(g)$. Without loss of generality $f \in A$. Then $\mathscr{U}(A)=\mathscr{U}(A \cup B)$ if $g \in A$, and $\mathscr{U}(A) \geq \mathscr{U}(A \cup B) \geq \mathscr{U}(B)$ if $g \in B$. However, $\succeq$ violates SSB: if $u_{1}(f)=0, u_{2}(f)=\frac{1}{2}, u_{1}\left(f^{\prime}\right)=1, u_{2}\left(f^{\prime}\right)=0, u_{1}(g)=0$, and $u_{2}(g)=1$, then $\{f\} \sim\left\{f, f^{\prime}\right\} \succ\left\{f^{\prime}\right\}$ but $\{f, g\} \succ\left\{f, f^{\prime}, g\right\}$.

A tuple $(u, p, Q, \kappa)$ as in Theorem 1 is said to represent $\succeq$. The representing tuple is unique (up to cardinal equivalence for $u$ ) if the degenerate case of strategic rationality is excluded. Thus it is meaningful to ask about behavioral interpretations of its components. We have already noted $u$ and $p: u$ ranks lotteries (constant acts) and $p$ is the 'commitment prior' - it underlies the ranking of singleton menus. Turn to $Q$ and $\kappa$. In what follows, we adopt variants of GP's comparative notions 'greater preference for commitment' and 'greater self-control,' renamed so as to reflect better the story we have in mind. Note that while the behavioral notions studied here are simple variants of those studied by GP, the characterization results to follow are not corollaries of GP's results, as a comparison of the respective proofs will reveal.

Say that $\succeq^{*}$ exhibits greater ex post pessimism than $\succeq$ if for all acts $f$ and $g$,

$$
\{f\} \succ\{f, g\} \Longrightarrow\{f\} \succ^{*}\{f, g\} .
$$

The ranking $\{f\} \succ\{f, g\}$ indicates that though $f$ is better than $g$ ex ante, the agent is tempted to choose $g$ ex post when holding the menu $\{f, g\}$. This reversal occurs because the agent with preference $\succeq$ becomes more pessimistic ex post about the prospects for the menu $\{f, g\}$. If $\succeq *$ exhibits greater pessimism ex post, then she should also strictly prefer $\{f\}$ to $\{f, g\}$. 
THEOREM 2. Suppose that both $\succeq$ and $\succeq^{*}$ have utility representations (1)-(3), with components $(u, p, Q, \kappa)$ and $\left(u^{*}, p^{*}, Q^{*}, \kappa^{*}\right)$ respectively, and that neither is strategically rational. Then $\succeq *$ exhibits greater ex post pessimism than $\succeq$ if and only if

$$
(u, p)=\left(a u^{*}+b, p^{*}\right) \text { for some } a>0 \text { and some } b
$$

and

$$
Q=(1-\epsilon)\{p\}+\epsilon Q^{*} \text {, for some } 0<\epsilon \leq 1 .
$$

The characterizing conditions assert both that the commitment rankings induced by $\succeq$ and $\succeq^{*}$ coincide (this is (6)) and that $Q$ is 'closer to $p$ ' than is $Q^{*}$ in the sense of an epsilon contamination (this is (7)). Since $Q^{*}$ is convex and contains $p$, (7) implies in particular that $Q \subseteq Q^{*}$, but it implies more.

Note that if $\succeq$ is strategically rational, then any $\succeq *$ exhibits greater ex post pessimism (the defining condition is satisfied vacuously) and no restrictions on commitment preferences are implied. If $\succeq^{*}$ is strategically rational, then (5) is satisfied if and only if $\succeq$ is also strategically rational, and again, condition (6) is not implied.

We are interested not only in how much pessimism an agent experiences (or expects to experience) ex post, but also in what she does about it, or more precisely, in the extent to which ex post choices are distorted by 'cold feet.' Say that $\beth^{*}$ is more panicky than $\succeq$ if it has greater ex post pessimism than $\succeq$ and

$$
\{f\} \succ\{f, g\} \sim\{g\} \Longrightarrow\{f\} \succ^{*}\{f, g\} \sim^{*}\{g\} .
$$

The hypothesized rankings for $\succeq$ indicate not only that the agent is tempted ex post to choose $g$ from the menu $\{f, g\}$, but also that she succumbs to this temptation, even though $f$ was optimal ex ante under commitment. She does this because she panics as the moment of truth approaches. If $\succeq^{*}$ is more panicky, then she should also choose $g$ out of $\{f, g\}$.

THEOREM 3. Suppose that both $\succeq$ and $\succeq^{*}$ have utility representations (1)-(3), with components $(u, p, Q, \kappa)$ and $\left(u^{*}, p^{*}, Q^{*}, \kappa^{*}\right)$ respectively, and that neither is strategically rational. Then $\succeq^{*}$ is more panicky than $\succeq$ if and only if $\left(u^{*}, p^{*}, Q^{*}, \kappa^{*}\right)$ and $(u, p, Q, \kappa)$ satisfy (6), (7), and $\kappa^{*} /\left(1+\kappa^{*}\right) \geq \epsilon \kappa /(1+\kappa)$.

It follows that $\succeq^{*}$ and $\succeq$ are equally pessimistic ex post (each has greater ex post pessimism than the other) but that $\succeq *$ is more panicky than $\succeq$ if and only if $(u, p, Q)=$ $\left(a u^{*}+b, p^{*}, Q^{*}\right)$ and $\kappa^{*} \geq \kappa$.

\section{CONCLUDING REMARKS}

To conclude, we describe a variation of the above model, and then an extension.

\subsection{Optimism}

Just as the Gilboa-Schmeidler model has a counterpart, where minimization over the set of priors is replaced by maximization, that captures ambiguity loving, so too a variation 
of our model can be used to model ex post optimism. The individual we have in mind is one who becomes more optimistic at the ex post stage in order to feel better about her available options. For example, Aronson (1995, pp. 183-5) refers to the psychology of inevitability whereby people attempt to 'make the best of things.'

Such an individual behaves as though she chooses beliefs ex post so as to make her menu attractive, and thus is captured by our utility functional form if in (3), $\min _{q \in Q}$ is replaced by $\max _{q \in Q}$. At the axiomatic level, only the last two axioms must be modified. Replace Constants-cannot-be-tempted by Constants-Do-Not-Tempt: For all $c \in \mathscr{C}$ and $f \in \mathscr{H}$,

$$
\{f\} \succ\{c\} \Longrightarrow\{f\} \sim\{c, f\}
$$

and replace Concave Temptation by Convex Temptation, which requires that $\{f \in \mathscr{H}$ : $\{c\} \sim\{c, f\} \succ\{f\}\}$ be convex for every $c \in \mathscr{C}$. These new axioms are readily interpreted along the lines of the interpretations provided for the original axioms. Finally, with these modifications, Theorem 1 is valid.

\subsection{Response to information}

The loss of confidence as the moment of truth approaches would presumably affect the way in which an individual responds to information-one would expect signals received ex post to be interpreted pessimistically. By adding a signal realized at time 1 and building on Epstein (2006), we can extend our model to capture also the response to information. ${ }^{14}$

An outline of the model follows. Let $S_{1}$ denote the (finite) space of signals, one of which is realized at time 1 . Ex ante, the agent chooses a contingent menu-a mapping $F$ from signals into menus of Anscombe-Aumann acts. At time 1, she observes the realized signal, updates her beliefs about $S$, and then chooses an act from the realized menu $F\left(s_{1}\right)$. Denote by $p$ prior beliefs on $S_{1} \times S$, by $p_{1}$ its first marginal, and, for each signal $s_{1}$, let $Q_{s_{1}}$ be a (closed and convex) set of probability measures on $S$ containing $p\left(\cdot \mid s_{1}\right)$, the Bayesian update of $p$. Then the utility of any contingent menu $F$ is given by

$$
\mathscr{W}(F)=\int_{S_{1}} \mathscr{U}\left(F\left(s_{1}\right) ; s_{1}\right) d p_{1}\left(s_{1}\right),
$$

where, for any menu $A$,

$$
\begin{gathered}
\mathscr{U}\left(A ; s_{1}\right)=\max _{h \in A}\left[U\left(h ; s_{1}\right)+\kappa V\left(h ; s_{1}\right)\right]-\kappa \max _{h^{\prime} \in A} V\left(h^{\prime} ; s_{1}\right) \\
U\left(h ; s_{1}\right)=p\left(\cdot \mid s_{1}\right) \cdot u(h) \\
V\left(h ; s_{1}\right)=\min _{q \in Q_{s_{1}}} q \cdot u(h) .
\end{gathered}
$$

The interpretation is clear given the parallel with our model (1)-(3). The key is that at the ex post stage, the agent does not rely simply on the Bayesian update $p\left(\cdot \mid s_{1}\right)$ of her prior

\footnotetext{
${ }^{14}$ We describe only the setup and the functional form for utility. However, it would be straightforward to provide foundations by suitably merging the two sets of axioms.
} 
beliefs, but rather behaves as though she adjusts the latter in a direction that renders the realized menu $F\left(s_{1}\right)$ unattractive, as indicated by the minimization over $Q_{s_{1}}$. In this way, 'cold feet' affect also her interpretation of the signal. (In Epstein's model, each set $Q_{s_{1}}$ is a singleton so that the updating bias does not depend on the realized menu.)

Similarly, an individual who tends to distort her beliefs ex post in order to feel better about her options would presumably bias her interpretation of a signal so that it is favorable to her options. Such updating is captured if $V$ is defined by a max rather than a min. The resulting bias resembles the so-called confirmatory bias, whereby people tend to interpret evidence in ways that confirm prior beliefs, as opposed to the menu in hand (see Rabin and Schragg 1999, for example).

\section{APPENDIX}

\section{A. Proof of the Main Representation Result, Theorem 1}

For necessity, verification of the axioms is straightforward.

The proof of sufficiency proceeds roughly as follows. Apply the Anscombe-Aumann Theorem to conclude that the preference $\succeq$ restricted to singleton menus $\{f\}$ has a unique expected utility representation

$$
U(f)=p \cdot u(f)
$$

where $p$ is a probability measure on $S$, and $u: \mathscr{C} \rightarrow \mathbb{R}$ is a linear utility index. Next, for any $f \in \mathscr{H}$, let

$$
\mathscr{H}_{f}=\{\alpha f+(1-\alpha) c: \alpha \in[0,1], c \in \mathscr{C}\},
$$

and let $\mathscr{A}_{f}$ be the class of menus in $\mathscr{H}_{f}$. Then $\mathscr{H}_{f}$ is a compact mixture space, and $\succeq$ restricted to $\mathscr{A}_{f}$ satisfies Independence (because $\succeq$ satisfies Collinear Independence) and Set-Betweenness. Kopylov (2005, Theorem 2.1) extends GP's main representation result (Theorem 1) for preferences over menus in abstract mixture spaces. By this extension, one obtains a continuous and linear function $V_{f}: \mathscr{H}_{f} \rightarrow \mathbb{R}$ such that

$$
\mathscr{U}(A)=\max _{h \in A}\left(U(h)+V_{f}(h)\right)-\max _{h \in A} V_{f}(h)
$$

represents $\succeq$ on $\mathscr{A}_{f}$. The critical step is to extend the local functions $V_{f}$ to a global temptation function $V$. The remaining step is to show that $V$ has the form (3) for some $Q$, which follows from Gilboa and Schmeidler (1989).

We turn to the detailed proof. Throughout we abbreviate the domain $\mathscr{K}(\mathscr{H})$ by $\mathscr{A}$, and assume that $\succeq$ is non-degenerate, that is, $A \succ B$ for some $A, B \in \mathscr{A}$. (Otherwise, the desired representation holds trivially with $u \equiv 0$.)

Fix lotteries $c_{+}, c_{-} \in \mathscr{C}$ such that $\left\{c_{+}\right\} \succeq\{c\} \succeq\left\{c_{-}\right\}$for all $c \in \mathscr{C}$. Such $c_{+}$and $c_{-}$exist because $\mathscr{C}$ is compact and $\succeq$ is continuous. By Monotonicity, $\left\{c_{+}\right\} \succeq\{f\} \succeq\left\{c_{-}\right\}$for all $f \in \mathscr{H}$. By SSB, $\left\{c_{+}\right\} \succeq A \succeq\left\{c_{-}\right\}$for all finite menus $A$; by Continuity, $\left\{c_{+}\right\} \succeq A \succeq\left\{c_{-}\right\}$for all menus $A \in \mathscr{A}$. As $\succeq$ is non-degenerate, $\left\{c_{+}\right\} \succ\left\{c_{-}\right\}$.

Let $U: \mathscr{H} \rightarrow \mathbb{R}$ be the unique expected utility function (8) normalized by $u\left(c_{+}\right)=1$ and $u\left(c_{-}\right)=-1$. 
Take any menu $A \in \mathscr{A}$. Note that $\left\{c_{+}\right\} \succeq A \succeq\left\{c_{-}\right\}$. By Continuity, there is a unique $\alpha \in[0,1]$ such that $A \sim\left\{\alpha c_{+}+(1-\alpha) c_{-}\right\}$. Let $e(A)=\alpha c_{+}+(1-\alpha) c_{-}$and

$$
\mathscr{U}(A)=u(e(A)) \text {. }
$$

Then $\mathscr{U}: \mathscr{A} \rightarrow \mathbb{R}$ is the unique function that represents $\succeq$ and satisfies $\mathscr{U}(\{f\})=U(f)$ for all $f \in \mathscr{H}$. Moreover, $\mathscr{U}$ is continuous because $\succeq$ satisfies Continuity and hence the sets $\{A \in \mathscr{A}: \mathscr{U}(A) \leq \gamma\}$ and $\{A \in \mathscr{A}: \mathscr{U}(A) \geq \gamma\}$ are closed for all $\gamma \in \mathbb{R}$.

Hereafter, let $c_{0}=\frac{1}{2}\left(c_{+}+c_{-}\right)$; then $u\left(c_{0}\right)=0$. For any act $f \in \mathscr{H}$, let

- $e(f)=e(\{f\})$ so that $e(f) \in \mathscr{C}$ and $\{f\} \sim\{e(f)\}$

- $f+\gamma=\gamma c_{+}+(1-\gamma) f$ and $f-\gamma=\gamma c_{-}+(1-\gamma) f$ for $\gamma \in[0,1]$

- $f+\gamma=f-(-\gamma)$ and $f-\gamma=f+(-\gamma)$ for $\gamma \in[-1,0]$.

Note that

$$
f-\frac{U(f)}{1+|U(f)|}= \begin{cases}\frac{-U\left(c_{-}\right)}{-U\left(c_{-}\right)+U(f)} f+\frac{U(f)}{-U\left(c_{-}\right)+U(f)} c_{-} & \text {if } U(f) \geq 0 \\ \frac{U\left(c_{+}\right)}{U\left(c_{+}\right)-U(f)} f+\frac{-U(f)}{U\left(c_{+}\right)-U(f)} c_{+} & \text {if } U(f)<0\end{cases}
$$

and hence

$$
U\left(f-\frac{U(f)}{1+|U(f)|}\right)=0 .
$$

Say that $f \in \mathscr{H}$ is temptable if $\succeq$ is not strategically rational on $\mathscr{A}_{f}$, that is, on the domain of all menus that consist of mixtures $\alpha f+(1-\alpha) c$ of the act $f$ with constant acts $c$. The following lemma pins down the structure of the utility function $\mathscr{U}$ on the subspace $\mathscr{A}_{f}$.

LEMma 1. If $f \in \mathscr{H}$ is temptable, then there is a unique triple $\left(V_{f}, W_{f}, \kappa_{f}\right)$ such that

(i) $V_{f}, W_{f}: \mathscr{H}_{f} \rightarrow \mathbb{R}$ are continuous linear functions, and for all menus $A \in \mathscr{A}_{f}$,

$$
\mathscr{U}(A)=\max _{g \in A} W_{f}(g)-\max _{g \in A} V_{f}(g)
$$

(ii) $W_{f}(g)=U(g)+V_{f}(g)$ for all $g \in \mathscr{H}_{f}$

(iii) $\kappa_{f}>0$ and $V_{f}(c)=\kappa_{f} U(c)$ for all $c \in \mathscr{C}$.

Moreover, $\kappa_{f}=\kappa_{g}$ for any other temptable act $g \in \mathscr{H}$.

Proof. Fix a temptable act $f \in \mathscr{H}$ and invoke Kopylov (2005, Theorem 2.1): $\mathscr{H}_{f}$ is a compact mixture space satisfying properties M1-M4 in Kopylov (2005), and $\succeq$ restricted 
to $\mathscr{A}_{f}$ satisfies Order, Continuity, Binary Independence, and Set-Betweenness, the axioms in the cited theorem. Thus $\succeq$ can be represented on $\mathscr{A}_{f}$ by

$$
\mathscr{U}_{f}(A)=\max _{g \in A}\left(U_{f}(g)+V_{f}(g)\right)-\max _{g \in A} V_{f}(g)
$$

where $U_{f}, V_{f}: \mathscr{H}_{f} \rightarrow \mathbb{R}$ are continuous linear functions normalized by $U_{f}\left(c_{+}\right)=1$ and $U_{f}\left(c_{0}\right)=V_{f}\left(c_{0}\right)=0$. Then $U_{f}(\cdot)=u(\cdot)$ on $\mathscr{C}$ because both $U_{f}$ and $u$ are linear on $\mathscr{C}$, have equivalent normalizations, and represent the same ranking of lotteries. For any menu $A$ in $\mathscr{A}_{f}, \mathscr{U}(A)=u(e(A))=\mathscr{U}_{f}(e(A))=\mathscr{U}_{f}(A)$. Thus $\mathscr{U}_{f}(\cdot)=\mathscr{U}(\cdot)$ on $\mathscr{A}_{f}$ and $U_{f}(\cdot)=U(\cdot)$ on $\mathscr{H}_{f}$. For all $g \in \mathscr{H}_{f}$, let $W_{f}(g)=U(g)+V_{f}(g)$. Then the representation (10) holds.

We now show that $V_{f}$ is monotonic. Take any $h, h^{\prime} \in \mathscr{H}_{f}$ such that $h$ dominates $h^{\prime}$. For all $\gamma \in(0,1)$, Monotonicity and the representation (8) imply $\{h+\gamma\} \sim\left\{h+\gamma, h^{\prime}-\gamma\right\} \succ$ $\left\{h^{\prime}-\gamma\right\}$ and hence $V_{f}(h+\gamma) \geq V_{f}\left(h^{\prime}-\gamma\right)$. Let $\gamma \rightarrow 0$; then $V_{f}(h) \geq V_{f}\left(h^{\prime}\right)$ by continuity.

Let $\kappa_{f}=V_{f}\left(c_{+}\right)$. By linearity, $V_{f}\left(c_{-}\right)=-\kappa_{f}$. As $V_{f}$ is non-constant and monotonic, we have $\kappa_{f}>0$. For all $c \in \mathscr{C}$, the equality

$$
V_{f}(c)=V_{f}\left(\frac{1}{2}(1+U(c)) c_{+}+\frac{1}{2}(1-U(c)) c_{-}\right)=\kappa_{f} U(c)
$$

follows from the indifference $c \sim \frac{1}{2}(1+U(c)) c_{+}+\frac{1}{2}(1-U(c)) c_{-}$and the linearity of $V_{f}$.

Next, we show by contradiction that

$$
V_{f}(f)<\kappa_{f} U(f)
$$

If $V_{f}(f)>\kappa_{f} U(f)=V_{f}(e(f))$, then by continuity there is $\gamma$ such that $V_{f}(f-\gamma)>$ $V_{f}(e(f)+\gamma)$. By $(10),\{e(f)+\gamma\} \succ\{f-\gamma, e(f)+\gamma\} \succeq\{f-\gamma\}$, which contradicts Constantscannot-be-tempted (here the constant is $e(f)+\gamma$ ). If $V_{f}(f)=\kappa_{f} U(f)$, then for any $g=\alpha f+(1-\alpha) c \in \mathscr{H}_{f}$,

$$
V_{f}(g)=\alpha V_{f}(f)+(1-\alpha) V_{f}(c)=\kappa_{f}(\alpha U(f)+(1-\alpha) U(c))=\kappa_{f} U(g) .
$$

Therefore, $\mathscr{U}(A)=\max _{g \in A}\left(U(g)+V_{f}(g)\right)-\max _{g \in A} V_{f}(g)=\max _{g \in A} U(g)$ for all $A \in \mathscr{A}_{f}$. Thus $\succeq$ is strategically rational on $\mathscr{A}_{f}$, which contradicts $f$ being temptable.

Next we claim that for any sufficiently small $\alpha>0$, there is an act $f_{*} \in \mathscr{H}_{f}$ such that

$$
U\left(f_{*}\right)=2 \alpha>W_{f}\left(f_{*}\right)=\alpha>0>V_{f}\left(f_{*}\right)=-\alpha .
$$

To construct the required $f_{*}$, let $f_{+}=f-V_{f}(f) /\left(\kappa_{f}+\left|V_{f}(f)\right|\right)$ and $f_{-}=f-U(f) /(1+|U(f)|)$. By (9), $U\left(f_{-}\right)=0$. Similarly, $V_{f}\left(f_{+}\right)=0$ because $V_{f}$ is linear and satisfies $V_{f}\left(c_{+}\right)=\kappa_{f}$ and $V_{f}\left(c_{-}\right)=-\kappa_{f}$. By (11), $\kappa_{f} U\left(f_{+}\right)>V_{f}\left(f_{+}\right)$and $\kappa_{f} U\left(f_{-}\right)>V_{f}\left(f_{-}\right)$. Therefore $U\left(f_{+}\right)>0>$ $V_{f}\left(f_{-}\right)$. It follows that for sufficiently small $\alpha>0$, the mixture

$$
f_{*}=\frac{2 \alpha}{U\left(f_{+}\right)} f_{+}+\frac{\alpha}{-V_{f}\left(f_{-}\right)} f_{-}+\left(1-\frac{2 \alpha}{U\left(f_{+}\right)}-\frac{\alpha}{-V_{f}\left(f_{-}\right)}\right) c_{0}
$$

is well-defined and satisfies (12). 
By (10) and (12), $\left\{f_{*}\right\} \succ\left\{f_{*}, c_{0}\right\} \succ\left\{c_{0}\right\}$. By Kopylov (2005, Theorem 2.1), this ranking implies that $V_{f}$ and $W_{f} \equiv U+V_{f}$ are the unique functions that satisfy the representation (10), $W_{f}\left(c_{+}\right)=1$, and $W_{f}\left(c_{0}\right)=V_{f}\left(c_{0}\right)=0$. Clearly, $\kappa_{f}=V_{f}\left(c_{+}\right)$is also unique.

Finally, fix another temptable act $g$ and show that $\kappa_{f}=\kappa_{g}$. As in the argument for (12), for any sufficiently small $\alpha>0$, there is $g_{*} \in \mathscr{H}_{\text {g }}$ such that

$$
U\left(g_{*}\right)=2 \alpha>W_{g}\left(g_{*}\right)=\alpha>0>V_{g}\left(g_{*}\right)=-\alpha .
$$

By (10), $\left\{f_{*}\right\} \sim\left\{g_{*}\right\} \succ\left\{c_{0}, f_{*}\right\} \sim\left\{c_{0}, f_{*}, g_{*}\right\} \sim\left\{c_{0}, g_{*}\right\} \succ\left\{c_{0}\right\}$. By SSB,

$$
\left\{c_{0}, f_{*}\right\} \cup B \sim\left\{c_{0}, f_{*}, g_{*}\right\} \cup B \sim\left\{c_{0}, g_{*}\right\} \cup B
$$

for all menus $B \in \mathscr{A}$, and in particular, $\left\{c_{0}, f_{*}, c_{0}+\gamma\right\} \sim\left\{c_{0}, g_{*}, c_{0}+\gamma\right\}$. Take $\gamma>0$ such that $W_{f}\left(c_{0}+\gamma\right)<W_{f}\left(f_{*}\right)$ and $W_{g}\left(c_{0}+\gamma\right)<W_{g}\left(g_{*}\right)$. Then by (10),

$$
W_{f}\left(f_{*}\right)-V_{f}\left(c_{0}+\gamma\right)=\mathscr{U}\left(\left\{c_{0}, f_{*}, c_{0}+\gamma\right\}\right)=\mathscr{U}\left(\left\{c_{0}, g_{*}, c_{0}+\gamma\right\}\right)=W_{g}\left(g_{*}\right)-V_{g}\left(c_{0}+\gamma\right) .
$$

Thus $V_{f}\left(c_{0}+\gamma\right)=V_{g}\left(c_{0}+\gamma\right)$ and hence $\kappa_{f}=\kappa_{g}$.

Fix $\kappa>0$ such that $\kappa_{f}=\kappa$ for all temptable acts $f \in \mathscr{H}$. Such a value of $\kappa$ exists by Lemma 1. Moreover, if there are temptable acts $f \in \mathscr{H}$, then $\kappa$ is unique; otherwise, $\kappa$ is arbitrary.

For every act $g \in \mathscr{H}$ and menu $A \in \mathscr{A}$, let

$$
\begin{aligned}
& V(g)= \begin{cases}V_{g}(g) & \text { if } g \text { is temptable } \\
\kappa U(g) & \text { if } g \text { is not temptable }\end{cases} \\
& W(g)=U(g)+V(g)= \begin{cases}U(g)+V_{g}(g) & \text { if } g \text { is temptable } \\
(\kappa+1) U(g) & \text { if } g \text { is not temptable }\end{cases} \\
& \mathscr{U}_{W V}(A)=\max _{g \in A} W(g)-\max _{g \in A} V(g) .
\end{aligned}
$$

Later we show that $V$ is continuous and hence the maxima in the definition of $\mathscr{U}_{W V}$ are well-defined even if $A$ is not finite.

The following lemma asserts that the function $\mathscr{U}_{W V}$ extends the representation (10) to arbitrary finite menus.

LEMMA 2. For all finite menus $A, \mathscr{U}(A)=\mathscr{U}_{W V}(A)$.

ProOF. To prove this lemma, consider several cases.

Case 1: $A \in \mathscr{A}_{f}$ and $f \in \mathscr{H}$ is not temptable. Then all acts $g \in \mathscr{H}_{f}$ are not temptable because $\succeq$ is strategically rational on $\mathscr{H}_{g} \subseteq \mathscr{H}_{f}$. Accordingly, $V(\cdot)=\kappa U(\cdot)$ and $W(\cdot)=$ $(1+\kappa) U(\cdot)$ on $\mathscr{H}_{f}$. By strategic rationality,

$$
\mathscr{U}(A)=\max _{g \in A} U(g)=\max _{g \in A}(U(g)+\kappa U(g))-\max _{g \in A} \kappa U(g)=\mathscr{U}_{W V}(A) .
$$


Case 2: $A \in \mathscr{A}_{f}$ and $f \in \mathscr{H}$ is temptable. Take any $g=\alpha f+(1-\alpha) c \in \mathscr{H}_{f}$. If $\alpha=0$, then $g=c$ is not temptable, and $V(c)=\kappa U(c)=\kappa_{f} U(c)=V_{f}(c)$. If $\alpha>0$, then $g$ is temptable because a violation of strategic rationality on $\mathscr{A}_{f}$ can be translated into a similar violation on $\mathscr{A}_{g}$ by Collinear Independence. The function $V_{f}$ satisfies the representation (10) restricted to $\mathscr{H}_{g} \subseteq \mathscr{H}_{f}$. As $V_{g}$ is unique, $V(g)=V_{g}(g)=V_{f}(g)$. It follows that $V(\cdot)=V_{f}(\cdot)$ and $W(\cdot)=W_{f}(\cdot)$ on $\mathscr{H}_{f}$. By $(10)$,

$$
\mathscr{U}(A)=\max _{g \in A} W_{f}(g)-\max _{g \in A} V_{f}(g)=\max _{g \in A} W(g)-\max _{g \in A} V(g)=\mathscr{U}_{W V}(A) .
$$

Note that both $V(\cdot)=\kappa U(\cdot)$ on $\mathscr{H}_{f}$ in Case 1 and $V(\cdot)=V_{f}(\cdot)$ on $\mathscr{H}_{f}$ in Case 2 satisfy $C$-independence. In other words, for all $f \in \mathscr{H}, c \in \mathscr{C}$, and $\gamma \in[0,1]$,

$$
V(\gamma f+(1-\gamma) c)=\gamma V(f)+(1-\gamma) V(c)
$$

Similarly, $W$ is C-independent.

Case 3: $A=\{f, g\}$ and $f$ is not temptable. Then for all $\gamma \in(0,1)$,

$$
\begin{aligned}
& \{f+\gamma\} \sim\{f+\gamma, e(f)-\gamma\} \succ\{e(f)-\gamma\} \\
& \{e(f)+\gamma\} \sim\{e(f)+\gamma, f-\gamma\} \succ\{f-\gamma\}
\end{aligned}
$$

because $\succeq$ is strategically rational on $\mathscr{A}_{f}$. By SSB,

$$
\begin{aligned}
& \{f+\gamma\} \cup\{g\} \sim\{f+\gamma, e(f)-\gamma\} \cup\{g\} \\
& \{e(f)+\gamma\} \cup\{g\} \sim\{e(f)+\gamma, f-\gamma\} \cup\{g\} .
\end{aligned}
$$

Let $\gamma \rightarrow 0$; by Continuity $\{f, g\} \sim\{f, e(f), g\} \sim\{e(f), g\}$. By Cases 1 and 2 , as $V(f)=V(e(f)), W(f)=W(e(f))$ and $\{e(f), g\} \in \mathscr{A}_{g}$, we have

$$
\mathscr{U}(\{f, g\})=\mathscr{U}(\{e(f), g\})=\mathscr{U}_{W V}(\{e(f), g\})=\mathscr{U}_{W V}(\{f, g\}) .
$$

Case 4: $A=\{f, g\}$ and both $f$ and $g$ are temptable. If $U(f)=U(g)$, then $U(\{f, g\})=$ $\mathscr{U}_{W V}(\{f, g\})$ because $U(\{f, g\})=U(f)$ by SSB and $\mathscr{U}_{W V}(\{f, g\})=U(f)$ by direct verification. Assume without loss of generality that $U(f)>U(g)$ and consider two subcases.

Subcase 4.1: $V(g) \geq V(f)$. The inequalities $V\left(c_{-}\right) \leq V(f) \leq V(g) \leq V\left(c_{+}\right)$and Cindependence (13) imply that there are $c_{f}, c_{g} \in \mathscr{C}$ such that $V\left(c_{f}\right)=V(f)$ and $V\left(c_{g}\right)=V(g)$. Take $f^{\prime} \in \mathscr{H}_{f}$ such that $U\left(f^{\prime}\right)=U(g)$ and $V\left(f^{\prime}\right)=V(g)$. The construction of $f^{\prime}$ is illustrated in Figure 1 where acts $h \in \mathscr{H}$ are projected into points $(U(h), V(h))$ in the coordinate plane. Then (i) the segment connecting the points $(-1,-\kappa)$ and $(1, \kappa)$ consists of the projections of constant acts $c \in \mathscr{C}$, (ii) the projections of the temptable acts $f$ and $g$ lie strictly below this segment, and (iii) the projection of $g$ lies in the triangle formed 


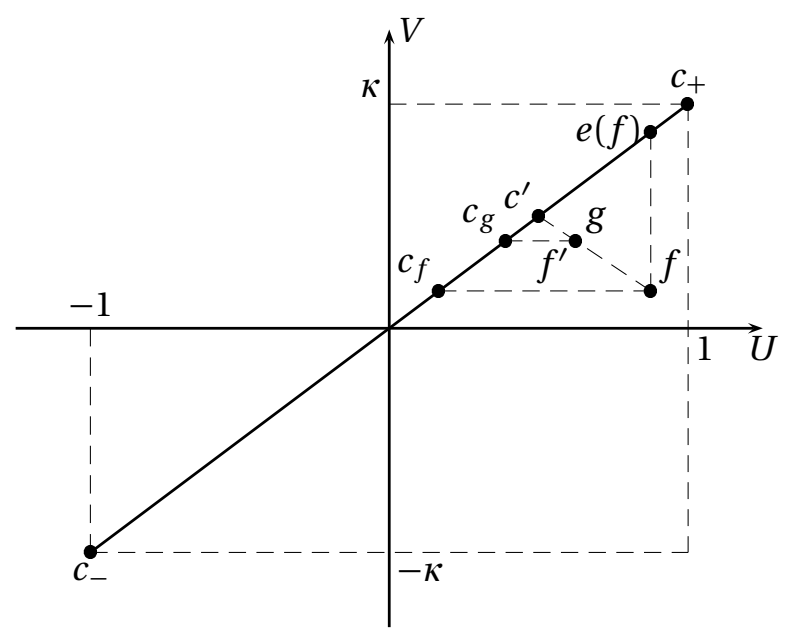

FIGURE 1. Subcase 4.1.

by the projections of $f, c_{f}$, and $e(f)$ because $U(g)<U(f)$ and $V(g) \geq V(f)$. It is clear that there exist $\alpha>0$ and $c^{\prime} \in \mathscr{C}$ such that

$$
(U(g), V(g))=\alpha(U(f), V(f))+(1-\alpha)\left(U\left(c^{\prime}\right), V\left(c^{\prime}\right)\right) .
$$

By C-Independence (13), $f^{\prime}=\alpha f+(1-\alpha) c$ satisfies $U\left(f^{\prime}\right)=U(g)$ and $V\left(f^{\prime}\right)=$ $V(g)$.

Note that $V(g)=V\left(c_{g}\right)$ implies $U(g)>U\left(c_{g}\right)$ because $g$ is temptable. Therefore, $W(g)=W\left(f^{\prime}\right)>W\left(c_{g}\right)$. Take any small $\gamma>0$ such that $W\left(f^{\prime}-\gamma\right)>$ $W\left(c_{g}+\gamma\right)$ and $W(g-\gamma)>W\left(c_{g}+\gamma\right)$. As $U, V$, and $W$ are C-independent, by Case 2,

$$
\begin{gathered}
\left\{f^{\prime}+\gamma\right\} \sim\left\{f^{\prime}+\gamma, c_{g}+\gamma\right\} \succ\left\{c_{g}+\gamma\right\} \\
\left\{f^{\prime}-\gamma\right\} \sim\{g-\gamma\} \succ\left\{c_{g}+\gamma, g-\gamma\right\} \sim\left\{c_{g}+\gamma, g-\gamma, f^{\prime}-\gamma\right\} \sim\left\{c_{g}+\gamma, f^{\prime}-\gamma\right\} .
\end{gathered}
$$

SSB implies that for all menus $B$,

$$
\begin{gathered}
\left\{f^{\prime}+\gamma\right\} \cup B \sim\left\{f^{\prime}+\gamma, c_{g}+\gamma\right\} \cup B \\
\left\{c_{g}+\gamma, g-\gamma\right\} \cup B \sim\left\{c_{g}+\gamma, g-\gamma, f^{\prime}-\gamma\right\} \cup B \sim\left\{c_{g}+\gamma, f^{\prime}-\gamma\right\} \cup B .
\end{gathered}
$$

Take $B=\left\{f^{\prime}+\gamma\right\}$. Then

$$
\left\{f^{\prime}+\gamma, g-\gamma\right\} \sim\left\{f^{\prime}+\gamma, c_{g}+\gamma, g-\gamma\right\} \sim\left\{f^{\prime}+\gamma, c_{g}+\gamma, f^{\prime}-\gamma\right\} \sim\left\{f^{\prime}+\gamma\right\} .
$$

Thus $\left\{f^{\prime}+\gamma\right\} \sim\left\{f^{\prime}+\gamma, g-\gamma\right\} \succ\{g-\gamma\}$. By SSB, for all menus $B$,

$$
\left\{f^{\prime}+\gamma\right\} \cup B \sim\left\{f^{\prime}+\gamma, g-\gamma\right\} \cup B
$$




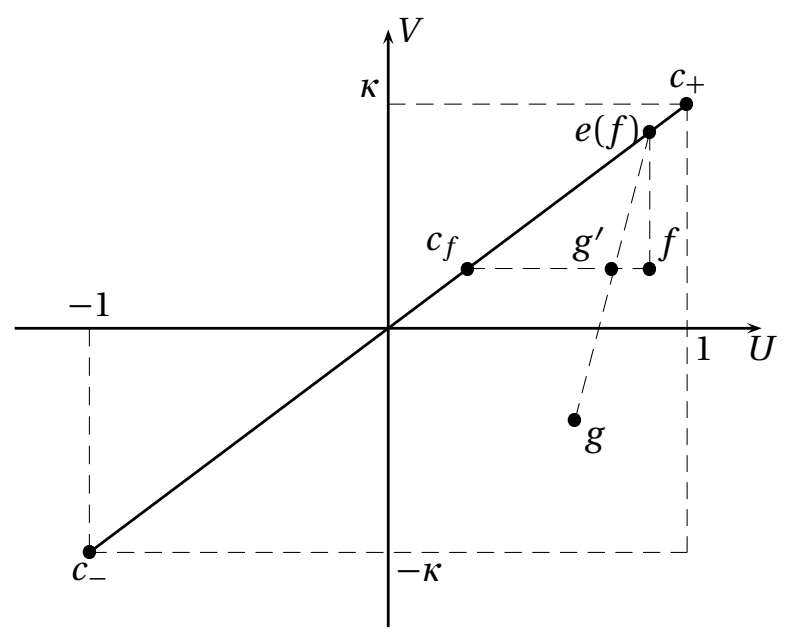

FIGURE 2. Subcase 4.2.

and similarly

$$
\{g+\gamma\} \cup B \sim\left\{g+\gamma, f^{\prime}-\gamma\right\} \cup B .
$$

By taking $B=\{f\}$ and $\gamma \rightarrow 0$, we conclude that $\left\{f^{\prime}, f\right\} \sim\left\{f^{\prime}, g, f\right\} \sim\{g, f\}$. Thus

$$
\mathscr{U}(\{f, g\})=\mathscr{U}\left(\left\{f, f^{\prime}\right\}\right)=\mathscr{U}_{W V}\left(\left\{f, f^{\prime}\right\}\right)=\mathscr{U}_{W V}(\{f, g\}) .
$$

Subcase 4.2: $V(g)<V(f)$. Then there is a mixture $g^{\prime}=\alpha g+(1-\alpha) e(f)$ such that $V\left(g^{\prime}\right)=V(f)$ and $U(f)>U\left(g^{\prime}\right)>U(g)$. The construction of $g^{\prime}$ is illustrated in Figure 2. Then by Subcase 4.1, $\mathscr{U}\left(\left\{f, g^{\prime}\right\}\right)=\mathscr{U}_{W V}\left(\left\{f, g^{\prime}\right\}\right)=U(f)>U\left(g^{\prime}\right)$, that is, $\{f\} \sim\left\{f, g^{\prime}\right\} \succ\left\{g^{\prime}\right\}$. Moreover, by Case $2,\left\{g^{\prime}\right\} \sim\left\{g, g^{\prime}\right\} \succ\{g\}$. By SSB, $\{f, g\} \sim\left\{f, g^{\prime}, g\right\} \sim\left\{f, g^{\prime}\right\} \sim\{f\}$. Thus $\mathscr{U}(\{f, g\})=U(f)=\mathscr{U}_{W V}(\{f, g\})$.

Case 5: $A$ is an arbitrary finite menu. Take $g_{A}, h_{A} \in A$ that deliver maxima on $A$ to the functions $W$ and $V$ respectively. Then for all $f \in A, W\left(g_{A}\right) \geq W(f), V\left(h_{A}\right) \geq V(f)$, and

$$
\mathscr{U}_{W V}\left(\left\{g_{A}, f\right\}\right) \geq \mathscr{U}_{W V}\left(\left\{g_{A}, h_{A}\right\}\right) \geq \mathscr{U}_{W V}\left(\left\{f, h_{A}\right\}\right) .
$$

Cases 3 and 4 imply that $\mathscr{U}\left(\left\{g_{A}, f\right\}\right) \geq \mathscr{U}\left(\left\{g_{A}, h_{A}\right\}\right) \geq \mathscr{U}\left(\left\{f, h_{A}\right\}\right)$, that is, $\left\{g_{A}, f\right\} \succeq$ $\left\{g_{A}, h_{A}\right\} \succeq\left\{f, h_{A}\right\}$. SSB implies, by induction with respect to the size of the set $A$, that

$$
A=\bigcup_{f \in A}\left\{g_{A}, f\right\} \succeq\left\{g_{A}, h_{A}\right\} \succeq \bigcup_{f \in A}\left\{f, h_{A}\right\}=A,
$$

that is, $A \sim\left\{g_{A}, h_{A}\right\}$. Thus, $\mathscr{U}(A)=\mathscr{U}\left(\left\{g_{A}, h_{A}\right\}\right)=\mathscr{U}_{W V}\left(\left\{g_{A}, h_{A}\right\}\right)=\mathscr{U}_{W V}(A)$.

The proof of the lemma is now complete.

The following lemma implies the desired structure for the function $V$. 
LEMMA 3. There exists a convex and closed set $Q$ of probability measures on $S$ such that for all $f \in \mathscr{H}$,

$$
V(f)=\kappa \min _{q \in Q} q \cdot u(f) .
$$

Moreover, $Q$ is unique and $p \in Q$.

Proof. The function $V$ is non-constant because $\kappa>0$, and satisfies C-independence (13). Next we show that $V$ is monotonic, continuous, and quasi-concave.

Monotonicity Take acts $f$ and $g$ such that $f$ dominates $g$. By Monotonicity, for all $\alpha \in$ $(0,1),\{f+\alpha\} \sim\{f+\alpha, g-\alpha\} \succ\{g-\alpha\}$. By Lemma $2, V(f+\alpha) \geq V(g-\alpha)$ and

$$
\alpha V\left(c_{+}\right)+(1-\alpha) V(f) \geq \alpha V\left(c_{-}\right)+(1-\alpha) V(g)
$$

because $V$ satisfies C-independence (13). Take $\alpha \rightarrow 0$ to deduce that $V(f) \geq V(g)$.

Continuity Let a sequence of acts $f_{n}$ converge to $f$ as $n \rightarrow \infty$. There exist sequences $\alpha_{n}$ and $\beta_{n}$ both converging to zero such that $f+\alpha_{n}$ dominates $f_{n}$, and $f_{n}$ dominates $f-\beta_{n}$. As $V$ is monotonic and C-independent,

$$
\alpha_{n} V\left(c_{+}\right)+\left(1-\alpha_{n}\right) V(f) \geq V\left(f_{n}\right) \geq \beta_{n} V\left(c_{-}\right)+\left(1-\beta_{n}\right) V(f) .
$$

It follows that $V(f)=\lim _{n \rightarrow \infty} V\left(f_{n}\right)$.

Quasi-concavity Suppose that $V(f)=V(g)>V(\alpha f+(1-\alpha) g)$. Take $c \in \mathscr{C}$ such that

$$
V(f)=V(g)>V(c)>V(\alpha f+(1-\alpha) g) .
$$

Then by (11), $V(e(f)) \geq V(f)>V(c)$ and $V(e(g)) \geq V(g)>V(c)$. By monotonicity of $V, U(f)>U(c), U(g)>U(c)$ and hence $U(\alpha f+(1-\alpha) g)>U(c)$. By Lemma 2, $\{f\} \sim\{f, c\} \succ\{c\}$ and $\{g\} \sim\{g, c\} \succ\{c\}$, but $\{\alpha f+(1-\alpha) g\} \succ\{\alpha f+(1-\alpha) g, c\} \succeq$ $\{c\}$, contradicting Concave Temptation.

The preceding argument shows that the ranking on $\mathscr{H}$ represented by $V$ satisfies all the axioms of Gilboa and Schmeidler's 1989 multiple-priors model. Thus $V$ has the form (14) and $Q$ is unique. The inclusion $p \in Q$ follows from the fact that for all $f \in \mathscr{H}$, $V(f) \leq V(e(f))=p \cdot u(f)$.

To complete the proof of the theorem, we show that $\mathscr{U} \equiv \mathscr{U}_{W V}$ on all of $\mathscr{A}$. Take an arbitrary menu $A$ and a sequence of finite menus $A_{1}, A_{2}, \ldots$ that converges to $A$ in the Hausdorff metric. By Lemma $2, \mathscr{U}\left(A_{i}\right)=\mathscr{U}_{W V}\left(A_{i}\right)$ for all $i$. As the functions $\mathscr{U}, V$, and $W$ are continuous, we have $\mathscr{U}(A)=\mathscr{U}_{W V}(A)$.

To show the required uniqueness of $(u, p, \kappa, Q)$ in the representation (1)-(3), suppose that this tuple can be replaced by $\left(u^{\prime}, p^{\prime}, \kappa^{\prime}, Q^{\prime}\right)$. Then $p^{\prime}=p, u^{\prime}$ is a positive linear transformation of $u$, and hence $(u, p, \kappa, Q)$ can be replaced by $\left(u, p, \kappa^{\prime}, Q^{\prime}\right)$ as well. Note that if there are no temptable acts in $\mathscr{H}$, then $V$ is a positive linear transformation of $U$ and $\succeq$ is strategically rational. Thus, if $\succeq$ is not strategically rational, then there exist temptable acts, and Lemmas 1 and 3 imply that $\kappa=\kappa^{\prime}$, and $Q=Q^{\prime}$. 


\section{B. Proofs For Comparative Pessimism}

Proof of Theorem 2. Let $\succeq^{*}$ and $\succeq$ conform to our model with the corresponding tuples $\left(u^{*}, p^{*}, Q^{*}, \kappa^{*}\right)$ and $(u, p, Q, \kappa)$. For all vectors $a \in \mathbb{R}^{S}$, let

$$
Q \cdot a=\min _{q \in Q} q \cdot a \text { and } Q^{*} \cdot a=\min _{q \in Q^{*}} q \cdot a .
$$

Suppose that neither preference is strategically rational. Then $\kappa, \kappa^{*}>0$ and sufficiency of (6) and (7) is immediate:

$$
\begin{aligned}
\{f\} \succ\{f, g\} & \Rightarrow \quad[p \cdot u(f)>p \cdot u(g)] \text { and }[Q \cdot u(g)>Q \cdot u(f)] \quad \Rightarrow \\
& {\left[p^{*} \cdot u^{*}(f)>p^{*} \cdot u^{*}(g)\right] \text { and }\left[Q^{*} \cdot u^{*}(g)>Q^{*} \cdot u^{*}(f)\right] }
\end{aligned}
$$

For necessity, let ${ }^{*}$ exhibit greater pessimism than $\succeq$.

Lemma 4. $p=p^{*}$ and $u$ is a positive linear transformation of $u^{*}$.

Proof. Adopt the same notation $c_{+}, c_{-}, c_{0}, f+\gamma, f-\gamma$ as in the proof of the main representation result. Without loss of generality let $u\left(c_{+}\right)=1, u\left(c_{-}\right)=-1$, and $u\left(c_{0}\right)=$ $u^{*}\left(c_{0}\right)=0$.

Fix an act $f \in \mathscr{H}$ that is temptable for the preference $\succeq$. This act exists because $\succeq$ is not strategically rational. Without loss of generality $U(f)=0>V(f)$.

We first show that for all acts $g \in \mathscr{H}$,

$$
U(g)=0 \quad \Rightarrow \quad U^{*}(g) \geq 0 .
$$

Take any $g \in \mathscr{H}$ such that $U(g)=0$. If $g$ is temptable, then for sufficiently small $\gamma>0$, $\{g\} \succ\left\{g, c_{0}-\gamma\right\} \succeq\left\{c_{0}-\gamma\right\}$. Thus, $\{g\} \succ^{*}\left\{g, c_{0}-\gamma\right\} \succeq^{*}\left\{c_{0}-\gamma\right\}$ and by continuity, $U^{*}(g) \geq$ $u^{*}\left(c_{0}\right)=0$. If $g$ is not temptable, then $W(g)=V(g)=U(g)=0$. Fix any $\alpha>0$ and let $f_{\alpha}=\alpha f+(1-\alpha) c_{0}$. Then for all sufficiently small $\gamma>0,\left\{f_{\alpha}+\gamma\right\} \succ\left\{f_{\alpha}+\gamma, g\right\}$. Thus $\left\{f_{\alpha}+\gamma\right\} \succ^{*}\left\{f_{\alpha}+\gamma, g\right\}$ and $V^{*}(g)>V^{*}\left(f_{\alpha}+\gamma\right)$. As $\alpha$ and $\gamma$ can be taken to be arbitrarily small, $V^{*}(g) \geq V^{*}\left(c_{0}\right)$ and hence $U^{*}(g) \geq u^{*}\left(c_{0}\right)=0$.

We now show that for all acts $g \in \mathscr{H}$,

$$
U(g)=0 \quad \Rightarrow \quad U^{*}(g)=0 .
$$

By (15), if $c \in \mathscr{C}$ and $c \sim c_{0}$, then $c \succeq^{*} c_{0}$. Analogously, if $c \sim c_{0}$, then $c_{0} \succeq^{*} c$. Thus $u(c)=0$ implies $u^{*}(c)=0$. Take any $g \in \mathscr{H}$ such that $U(g)=0$. Let $h \in \mathscr{H}$ be such that $u\left(\frac{1}{2}(g+h)\right)=0$, that is, $\frac{1}{2}(g(s)+h(s)) \sim c_{0}$ for all $s \in S$. The act $h$ exists because the range of the function $u$ is $[-1,1]$. By Monotonicity, $U\left(\frac{1}{2}(g+h)\right)=u\left(c_{0}\right)=0$, and hence $U(h)=0$. By (15), $U^{*}(g) \geq 0$ and $U^{*}(h) \geq 0$. Note that $\frac{1}{2}(g(s)+h(s)) \sim^{*} c_{0}$ for all $s \in S$. By Monotonicity, $U^{*}\left(\frac{1}{2}(g+h)\right)=u^{*}\left(c_{0}\right)=0$. It follows that $U^{*}(g)=U^{*}(h)=0$.

We now show that $u$ is a positive linear transformation of $u^{*}$. By (16), $\{f\} \sim^{*}\left\{c_{0}\right\}$. Take $\gamma>0$ such that $\{f\} \succ\left\{f, c_{0}-\gamma\right\}$. Then $\{f\} \succ^{*}\left\{c_{0}-\gamma\right\}$, that is, $\left\{c_{0}\right\} \succ^{*}\left\{c_{0}-\gamma\right\}$. It follows that $\left\{c_{+}\right\} \succ^{*}\left\{c_{0}\right\} \succ^{*}\left\{c_{-}\right\}$. Normalize $u^{*}$ so that $u^{*}\left(c_{+}\right)=1$ and $u^{*}\left(c_{-}\right)=-1$. Fix 
any $c \in \mathscr{C}$. Let $c^{\prime}=\frac{1}{2}(1-u(c)) c_{+}+\frac{1}{2}(1+u(c)) c_{-}$. Then $u\left(\frac{1}{2}\left(c+c^{\prime}\right)\right)=0$ and by (16), $u^{*}\left(\frac{1}{2}\left(c+c^{\prime}\right)\right)=0$. Thus $u^{*}(c)=-u^{*}\left(c^{\prime}\right)=-\frac{1}{2}(1-u(c)) u^{*}\left(c_{+}\right)-\frac{1}{2}(1+u(c)) u^{*}\left(c_{-}\right)=u(c)$.

Finally, we show that $p=p^{*}$. By (16), for all $g \in \mathscr{H}$, if $p \cdot u(g)=0$, then $p^{*} \cdot u^{*}(g)=$ $p^{*} \cdot u(g)=0$. It follows that for all vectors $a \in[-1,1]^{S}, p \cdot a=0$ implies $p^{*} \cdot a=0$. Thus $p^{*}=\gamma p$ for some $\gamma \in \mathbb{R}$, and $p=p^{*}$ because both are probability measures.

To prove that $Q=(1-\epsilon)\{p\}+\epsilon Q^{*}$ for some $0<\epsilon \leq 1$, we adopt a method analogous to the one used by Kopylov (2007). Let $\mathbb{D}$ be the set of all points $a \in \mathbb{R}^{S}$ where the functions $Q \cdot a$ and $Q^{*} \cdot a$ are both differentiable. For every $a \in \mathbb{D}$, let

$$
q(a)=\nabla(Q \cdot a) \quad \text { and } \quad q^{*}(a)=\nabla\left(Q^{*} \cdot a\right)
$$

be the derivatives of $Q \cdot a$ and $Q^{*} \cdot a$ respectively. Then $q(a) \in Q$ and $q^{*}(a) \in Q^{*}$ are the unique probability measures in $Q$ and $Q^{*}$ respectively such that

$$
Q \cdot a=q(a) \cdot a \text { and } Q^{*} \cdot a=q^{*}(a) \cdot a \text {. }
$$

To show (17), take any $q \in Q$ such that $Q \cdot a=q \cdot a$. For all $b \in \mathbb{R}^{S}$ and $\delta \in \mathbb{R}$,

$$
Q \cdot a+\delta(q \cdot b)=q \cdot(a+\delta b) \geq Q \cdot(a+\delta b)=Q \cdot a+\delta(q(a) \cdot b)+o(\delta),
$$

and hence $q \cdot b=q(a) \cdot b$. Thus $q=q(a)$, and similarly for $q^{*}(a)$.

Let $\overrightarrow{1}=(1, \ldots, 1) \in \mathbb{R}^{S}$. The set $\mathbb{D}$ and the functions $q$ and $q^{*}$ are preserved under transformations $\alpha a+\gamma \overrightarrow{1}$ for $\alpha>0$ and $\gamma \in \mathbb{R}$. Indeed, for all $b \in \mathbb{R}^{S}$,

$$
Q \cdot b=\alpha\left(Q \cdot\left(\frac{b-\gamma \overrightarrow{1}}{\alpha}\right)\right)+\gamma .
$$

By the chain rule, $Q \cdot b$ is differentiable at $b=\alpha a+\gamma \overrightarrow{1}$ and $q(\alpha a+\gamma \overrightarrow{1})=q(a)$. Similarly, $Q^{*} \cdot b$ is differentiable at $b=\alpha a+\gamma \overrightarrow{1}$ and $q^{*}(\alpha a+\gamma \overrightarrow{1})=q^{*}(a)$. Thus $\alpha a+\gamma \overrightarrow{1} \in \mathbb{D}$.

The following lemma delivers the required $\epsilon$.

Lemma 5. There exists $\epsilon \in[0,1]$ such that $q(a)=\epsilon q^{*}(a)+(1-\epsilon) p$ for all $a \in \mathbb{D}$.

Proof. We claim that for all $a, b \in \mathbb{R}^{S}$,

$$
[p \cdot a>p \cdot b] \text { and }[Q \cdot b>Q \cdot a] \quad \Rightarrow \quad Q^{*} \cdot b>Q^{*} \cdot a .
$$

Fix any $a, b \in \mathbb{R}^{S}$ such that $p \cdot a>p \cdot b$ and $Q \cdot b>Q \cdot a$. Take $\alpha>0$ such that $|\alpha a(s)|$, $|\alpha b(s)| \leq 1$ for all $s \in S$. Then $\alpha a=u(f)$ and $\alpha b=u(g)$ for some $f, g \in \mathscr{H}$. (Here $u(f)$ and $u(g)$ are vectors in $\mathbb{R}^{S}$.) Then

$$
\begin{gathered}
{[p \cdot a>p \cdot b] \text { and }[Q \cdot b>Q \cdot a] \Rightarrow} \\
{[p \cdot u(f)>p \cdot u(g)] \text { and }[Q \cdot u(g)>Q \cdot u(f)] \quad \Rightarrow \quad\{f\} \succ\{f, g\} \quad \Rightarrow} \\
\{f\} \succ^{*}\{f, g\} \Rightarrow Q^{*} \cdot u(g)>Q^{*} \cdot u(f) \quad \Rightarrow \quad Q^{*} \cdot b>Q^{*} \cdot a .
\end{gathered}
$$


Next we claim that for any $b \in \mathbb{D}$, there is $\epsilon_{b} \in[0,1]$ such that $q(b)=\epsilon_{b} q^{*}(b)+$ $\left(1-\epsilon_{b}\right) p$. Suppose that for some $b$ no such $\epsilon_{b}$ exists. Let $x \in \mathbb{R}^{S}$ separate $q(b)$ from the segment $\left[q^{*}(b), p\right]$ so that $q^{*}(b) \cdot x>0, p \cdot x>0$, but $q(b) \cdot x<0$. Then for sufficiently small $\delta>0$,

$$
p \cdot(b+\delta x)>p \cdot b \quad \text { and } \quad Q \cdot b=q(b) \cdot b>q(b) \cdot(b+\delta x) \geq Q \cdot(b+\delta x),
$$

but also $Q^{*} \cdot(b+\delta x)=Q^{*} \cdot b+\delta\left(q^{*}(b) \cdot x\right)+o(\delta)>Q^{*} \cdot b$. This is a contradiction with (18) for $a=b+\delta x$.

To complete the proof of the lemma, we show that for all $a, b \in \mathbb{D}, \epsilon_{a}=\epsilon_{b}$. Take $a, b \in \mathbb{D}$ such that $q^{*}(a) \neq p$ and $q^{*}(b) \neq p$. (Note that if $q^{*}(a)=p$, then the equality $\epsilon_{a}=\epsilon_{b}$ can be satisfied because $\epsilon_{a}$ is arbitrary. Similarly, if $q^{*}(b)=p$.) As $q^{*}(a) \neq p$ and $p=p^{*} \in Q^{*}$, by (17) we have $Q^{*} \cdot a<p \cdot a$. Similarly, $Q^{*} \cdot b<p \cdot b$. Let

$$
a^{\prime}=\frac{a-(p \cdot a) \overrightarrow{1}}{p \cdot a-Q^{*} \cdot a} \quad \text { and } \quad b^{\prime}=\frac{b-(p \cdot b) \overrightarrow{1}}{p \cdot b-Q^{*} \cdot b} .
$$

Then $q^{*}\left(a^{\prime}\right)=q^{*}(a), q^{*}\left(b^{\prime}\right)=q^{*}(b)$,

$$
q\left(a^{\prime}\right)=q(a)=\epsilon_{a} q^{*}(a)+\left(1-\epsilon_{a}\right) p, \quad \text { and } \quad q\left(b^{\prime}\right)=q(b)=\epsilon_{b} q^{*}(b)+\left(1-\epsilon_{b}\right) p .
$$

By construction, $p \cdot a^{\prime}=p \cdot b^{\prime}=0, Q^{*} \cdot a^{\prime}=Q^{*} \cdot b^{\prime}=-1, Q \cdot a^{\prime}=-\epsilon_{a}$, and $Q \cdot b^{\prime}=-\epsilon_{b}$. Suppose that $\epsilon_{a} \neq \epsilon_{b}$; without loss of generality let $\epsilon_{a}>\epsilon_{b}$. Then for sufficiently small $r>0$

$$
p \cdot\left(a^{\prime}+\gamma \overrightarrow{1}\right)=\gamma>p \cdot b^{\prime} \quad \text { and } Q \cdot\left(a^{\prime}+\gamma \overrightarrow{1}\right)=-\epsilon_{a}+\gamma<-\epsilon_{b}=Q \cdot b^{\prime},
$$

but $Q^{*} \cdot\left(a^{\prime}+\gamma \overrightarrow{1}\right)=-1+\gamma>Q^{*} \cdot b^{\prime}$. This contradicts (18). Thus $\epsilon_{a}=\epsilon_{b}$.

We conclude that $Q \cdot a=\epsilon\left(Q^{*} \cdot a\right)+(1-\epsilon)(p \cdot a)$ for all $a \in \mathbb{D}$. By Rockafellar (1970, Theorem 25.5), the complement of the set $\mathbb{D}$ has measure zero. Thus $\mathbb{D}$ is dense. Then by continuity,

$$
Q \cdot a=\epsilon\left(Q^{*} \cdot a\right)+(1-\epsilon)(p \cdot a)=\left(\epsilon Q^{*}+(1-\epsilon) p\right) \cdot a
$$

for all $a \in \mathbb{R}^{S}$. It follows that $Q=\epsilon Q^{*}+(1-\epsilon) p$. As $\succeq$ is not strategically rational, we have $\epsilon>0$. This completes the proof of Theorem 2 .

Proof of Theorem 3. Let $\succeq^{*}$ and $\succeq$ conform to our model with the corresponding tuples $\left(u^{*}, p^{*}, Q^{*}, \kappa^{*}\right)$ and $(u, p, Q, \kappa)$. Suppose that neither preference is strategically rational.

Let $P=(1 /(1+\kappa))\{p\}+(\kappa /(1+\kappa)) Q$ and $P^{*}=\left(1 /\left(1+\kappa^{*}\right)\right)\left\{p^{*}\right\}+\left(\kappa^{*} /\left(1+\kappa^{*}\right)\right) Q^{*}$. The conditions (6), (7), and $\kappa^{*} /\left(1+\kappa^{*}\right) \geq \epsilon \kappa /(1+\kappa)$ imply

$$
P=(1-\theta)\{p\}+\theta P^{*} .
$$

where $\theta=\epsilon(\kappa /(1+\kappa)) /\left(\kappa^{*} /\left(1+\kappa^{*}\right)\right)$.

Sufficiency of these conditions now follows from

$$
\begin{aligned}
&\{f\} \succ\{f, g\} \sim\{g\} \Rightarrow[p \cdot u(f)>p \cdot u(g)] \text { and }[P \cdot u(g)>P \cdot u(f)] \Rightarrow \\
& {\left[p^{*} \cdot u^{*}(f)>p^{*} \cdot u^{*}(g)\right] \text { and }\left[P^{*} \cdot u^{*}(g)>P^{*} \cdot u^{*}(f)\right] \Rightarrow \quad\{f\} \succ^{*}\{f, g\} \sim^{*}\{g\} . }
\end{aligned}
$$


For necessity, let $\succeq^{*}$ be more panicky than $\succeq$. Then $\succeq^{*}$ exhibits greater pessimism and Theorem 3 implies (6) and (7). Moreover, for all $a, b \in \mathbb{R}^{S}$,

$$
[p \cdot a>p \cdot b] \text { and }[P \cdot a>P \cdot b] \quad \Rightarrow \quad P^{*} \cdot b>P^{*} \cdot a .
$$

To prove this claim, fix any $a, b \in \mathbb{R}^{S}$. Take $\alpha>0$ and $f, g \in \mathscr{H}$ such that $\alpha a=u(f)$ and $\alpha b=u(g)$. Then

$$
\begin{aligned}
& {[p \cdot a>p \cdot b] \text { and }[P \cdot a>P \cdot b] \quad \Rightarrow} \\
& {[p \cdot u(f)>p \cdot u(g)] \text { and }[P \cdot u(g)>P \cdot u(f)] \quad \Rightarrow \quad\{f\} \succ\{f, g\} \sim\{g\} \quad \Rightarrow} \\
& \{f\} \succ^{*}\{f, g\} \sim^{*}\{g\} \Rightarrow P^{*} \cdot u(g)>P^{*} \cdot u(f) \quad \Rightarrow \quad P^{*} \cdot b>P^{*} \cdot a .
\end{aligned}
$$

Use the condition (19) to replace $Q$ and $Q^{*}$ by $P$ and $P^{*}$ in Lemma 5 and obtain $0<$ $\theta \leq 1$ such that $P=(1-\theta)\{p\}+\theta P^{*}$. In particular, $P \subseteq P^{*}$ and therefore also

$$
\left(1-\epsilon \frac{\kappa}{1+\kappa}\right)\{p\}+\epsilon \frac{\kappa}{1+\kappa} Q^{*} \subseteq\left(1-\frac{\kappa^{*}}{1+\kappa^{*}}\right)\{p\}+\frac{\kappa^{*}}{1+\kappa^{*}} Q^{*} .
$$

As $Q^{*}$ is a nonsingleton, $\epsilon \kappa /(1+\kappa) \leq \kappa^{*} /\left(1+\kappa^{*}\right)$.

\section{REFERENCES}

Aliprantis, Charalambos D. and Kim C. Border (1994), Infinite Dimensional Analysis. Springer, Berlin. [234]

Armor, David A. and Shelley E. Taylor (2002), "When predictions fail: The dilemma of unrealistic optimism." In Heuristics and Biases: The Psychology of Intuitive Judgment (Thomas Gilovich, Dale Griffin, and Daniel Kahneman, eds.), 334-347, Cambridge University Press, Cambridge. [231]

Aronson, Elliot (1995), The Social Animal, 7th edition. W. H. Freeman, New York. [245]

Chew, Soo Hong (1983), "A generalization of the quasilinear mean with applications to the measurement of income inequality and decision theory resolving the Allais paradox." Econometrica, 51, 1065-1092. [242]

Dekel, Eddie, Barton L. Lipman, and Aldo Rustichini (2006), “Temptation-driven preferences.” Unpublished paper, Department of Economics, Boston University. [234, 240]

Epstein, Larry G. (2006), "An axiomatic model of non-Bayesian updating." Review of Economic Studies, 73, 413-436. [233, 245]

Epstein, Larry G., Massimo Marinacci, and Kyoungwon Seo (forthcoming), "Coarse contingencies and ambiguity." Theoretical Economics. [234]

Epstein, Larry G. and Martin Schneider (2003), “Recursive multiple-priors.” Journal of Economic Theory, 113, 1-31. [232] 
Ergin, Haluk and Todd Sarver (2005), "A unique costly contemplation representation." Unpublished paper, Department of Economics, Massachusetts Institute of Technology. [234]

Frederick, Shane, George Loewenstein, and Ted O'Donoghue (2002), “Time discounting and time preference: a critical review." Journal of Economic Literature, 40, 351-401. [232]

Gilboa, Itzhak and David Schmeidler (1989), "Maxmin expected utility with non-unique prior.” Journal of Mathematical Economics, 18, 141-153. [231, 246, 253]

Gilovich, Thomas, Margaret Kerr, and Victoria H. Medvec (1993), "Effect of temporal perspective on subjective confidence." Journal of Personality and Social Psychology, 64, 552-560. [231]

Gul, Faruk and Wolfgang Pesendorfer (2001), “Temptation and self-control.” Econometrica, 69, 1403-1435. [232, 239]

Kopylov, Igor (2005), “Temptations in general settings.” Unpublished paper, Department of Economics, University of California, Irvine. [233, 239, 246, 247, 249]

Kopylov, Igor (2007), “Subjective probability under objective ambiguity.” Unpublished paper, Department of Economics, University of California, Irvine. [255]

Kreps, David M. (1979), “A representation theorem for 'preference for flexibility'." Econometrica, 47, 565-577. [235]

Kreps, David M. (1988), Notes on the Theory of Choice. Westview Press, Boulder. [238, 240]

Kreps, David M. (1992), "Static choice in the presence of unforeseen contingencies." In Economic analysis of markets and games (Partha Dasgupta, Douglas Gale, Oliver Hart, and Eric Maskin, eds.), 258-281, MIT Press, Cambridge. [235]

Machina, Mark J. (1984), “Temporal risk and the nature of induced preferences.” Journal of Economic Theory, 33, 199-231. [237]

Noor, Jawwad (2006), “Menu-dependent self-control.” Unpublished paper, Department of Economics, Boston University. [234, 240]

Noor, Jawwad (2007), “Temptation, welfare and revealed preference.” Unpublished paper, Department of Economics, Boston University. [232, 236, 240]

Rabin, Matthew and Joel L. Schragg (1999), "First impressions matter: a model of confirmatory bias.” Quarterly Journal of Economics, 114, 37-82. [246]

Rockafellar, R. Tyrrell (1970), Convex Analysis. Princeton University Press, Princeton. [256] 
Schmeidler, David (1989), "Subjective probability and expected utility without additivity." Econometrica, 57, 571-587. [242]

Trope, Yaacov and Nira Liberman (2003), “Temporal construal.” Psychological Review, 110, 403-421. [231]

Submitted 2007-1-17. Final version accepted 2007-7-7. Available online 2007-7-7. 